\title{
12. \\ Die Reduktion der elliptischen Integrale in ihre kanonische Form.
}

\author{
(Von Herrn E. Heine.)
}

Seit langer Zeit kennt man die Formeln, durch welche das Integral

$$
\boldsymbol{H}=\int_{g}^{h} \frac{d x}{\sqrt{\alpha+\beta x+\gamma x^{2}+\delta x^{3}+\varepsilon x^{4}}}
$$

in die kanonische Form der elliptischen Integrale verwandelt werden kann, wenn aufser $y$ und $h$ auch die Constanten $\alpha, \beta$, etc. reell sind. Ein besonderer Fall, in dem diese Constanten imaginär waren, - derselbe welcher \$. 26 behandelt ist - wurde Veranlassung zu dieser Abhandlung, in welcher die Transformationsformeln für den Fall zusammengestellt sind, dafs $\alpha$, etc. beliebige imaginäre Gröfsen bedeuten, die besonderen Fälle eingeschlossen, dafs die Constanten sämmtlich oder theilweise reell oder rein imaginär werden. Es zeigt sich, dafs dann $\boldsymbol{H}$ in die Form

$$
\frac{u+i v}{M}
$$

zerlegt werden kann, wo $\boldsymbol{M}$ eine gewisse, von den gegebenen Constanten einfach abhängende Gröfse bezeichnet, wo ferner $u$ aus der Summe oder Differenz eines gewissen Vielfachen des ganzen elliptischen Integrals $\boldsymbol{K}$ und eines zweiten elliptischen Integrals in der kanonischen Form besteht, und $v$ wie $u$ zusammengesetzt ist, nur dafs die Integrale welche $v$ ausmachen den complementären Modulus zu dem in $u$ vorkommenden haben. Die eben erwähnten elliptischen Integrale haben sämmtlich reelle Grenzen, die positiv und kleiner als 1 sind, so dafs wenn der Modulus reell ist, unsere Aufgabe gelöst ist. Wird aber der Modulus imaginär, so kann man sie in Abelsche Integrale zerlegen. Die Formeln hierzu sind im $\$$. 18 zusammengestellt; sie sind der bekannten Abhandlung von Jacobi entlehnt.

Der Gang der Rechnung, welche anzustellen war, ergiebt sich leicht. Es lälst sich $\boldsymbol{H}$ mit Hülfe bekannter Transformationsformeln in das Product eines Factors $\frac{1}{M}$ und eines elliptischen Integrales $\boldsymbol{J}$ in der Hauptform zerJournal f. d. M. Bd. LIII. Heft 3. 
legen, dessen Modulus $\varkappa$, (oder wenn $*$ imaginär ist; die Norm von $\varkappa$ ) kleiner als 1 ist, dessen Grenzen aber imaginär sind. Man kann die Transformation so einrichten, dafs $*$ nicht rein imaginär, sondern entweder reell oder complex ist. Diese Umformungen findet man im zweiten Abschnitt \$. 19.

Das Integral $\boldsymbol{J}$ oder

$$
\int \frac{d z}{\sqrt{1-z^{2}} \sqrt{1-x^{2} z^{2}}}
$$

auf welches man so geführt wird, ist ein vollständig bestimmtes, indem $z$ ein gegebenes Intervall auf gegebene Art durchläuft, und auch die Zeichen der Quadratwurzeln bekannt sind, wenn nur das der Quadratwurzel, welche in $\boldsymbol{H}$ vorkommt, gehörig bestimmt ist.' Dazu reicht im Allgemeinen aus, dafs man das Zeichen derselben für irgend einen Werth von $x$ kennt.

Die Zerfällnng von $\boldsymbol{J}$ in $u+i v$ bildet ausschliefslich den Gegenstand der ersten Abtheilung. Nachdem im $\mathfrak{\$} .1$ die bekannten Additionsformeln der elliptischen Functionen zusammengestellt, und im $\$$. 2 durch andere ersetzt

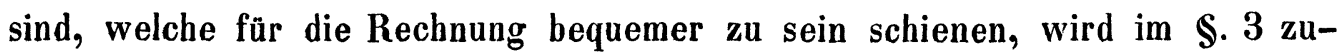
nächst darüber gehandelt, ob $\boldsymbol{J}$ einen bestimmten Werth hat. Darauf beginnt die Zerfällung und zwar zunächst für den Fall, dafs $\varkappa$ reell ist. Diese macht zwei verschiedene Untersuchungen nöthig; zuerst ist es nöthig, den Werth von $\sin a m u$ und $\sin a m v$ zu kennen, den bereits Richelot für ein reelles $x$ angegeben hat. Man findet ihn $\$$. 6 und $\$$. 10 , vereinfacht $\$$. 11. Die Anzahl von Vielfachen der Perioden, welche hinzuzufügen sind, die zweitens bestimmt werden mufs, ist $\$ .7-9$ und $\$$. $12-16$ aufgesucht. $\$ .5$ enthält eine häufig angewandte Transformationsformel, und $\$ .27$ ihre Verallgemeinerung. $\$$. $20-25$ ist ein interessanter specieller Fall behandelt, aus dem die Resultate des $\$ .26$ folgen.

\section{Abtheilung.}

\$. 1.

Das Fundament für unsere Untersuchungen bilden die bekannten Additionsformeln der elliptischen Functionen. Bezeichnet $*$ eine reelle oder imaginäre Gröfse, deren Modulus kleiner als 1 ist, sind ferner $\alpha, \beta, z$ reelle oder imàginäre Functionen einer reellen Gröfse $x$, welche durch die drei Gleichungen 


$$
\begin{aligned}
M_{z} & =\alpha \sqrt{1-\beta^{2}} \sqrt{1-\varkappa^{2} \beta^{2}}+\beta \sqrt{1-\alpha^{2}} \sqrt{1-\varkappa^{2} \alpha^{2}} \\
M_{\sqrt{1-z^{2}}} & =\sqrt{1-\alpha^{2}} \sqrt{1-\beta^{2}}-\alpha \beta \sqrt{1-\varkappa^{2} \alpha^{2}} \sqrt{1-\varkappa^{2} \beta^{2}} \\
M_{\sqrt{1-\varkappa^{2} z^{2}}} & =\sqrt{1-\varkappa^{2} \alpha^{2}} \sqrt{1-\varkappa^{2} \beta^{2}}-\varkappa^{2} \alpha \beta \sqrt{1-\alpha^{2}} \sqrt{1-\beta^{2}}
\end{aligned}
$$

zusammenhangen, in denen man zur Abkürzung

$$
\boldsymbol{M}=1-\varkappa^{2} \alpha^{2} \beta^{2}
$$

gesetzt hat, so wird

$$
\frac{\frac{d z}{d x}}{\sqrt{1-z^{2}} \sqrt{1-x^{2} z^{2}}}=\frac{\frac{d \alpha}{d x}}{\sqrt{1-\alpha^{2}} \sqrt{1-x^{2} \alpha^{2}}}+\frac{\frac{d \beta}{d x}}{\sqrt{1-\beta^{2}} \sqrt{1-x^{2} \beta^{2}}} .
$$

Denkt man sich z. B. $\alpha$ und $\beta$, so wie die Zeichen der Quadratwurzeln auf der rechten Seite gegeben, so bestimmt die erste Gleichung schon vollkommen den Werth von $z$, während die beiden anderen dazu dienen, die Zeichen von $\sqrt{1-z^{2}}$ und $\sqrt{1-\varkappa^{2} z^{2}}$ festzustellen, welche willkürlich angenommenen Zeichen der vier anderen Wurzelgröfsen entsprechen.

Obgleich diese Formeln unter der Voraussetzung bewiesen zu werden pflegen, dafs $\alpha, \beta, \varkappa$ reelle Gröfsen sind, so gelten sie bekanntlich noch für imaginäre Werthe derselben. Setzt man $\beta=\gamma i$, wo $\gamma$ wieder imaginär sein kann, und darauf $\gamma=\frac{\delta}{\sqrt{1-\delta^{2}}}$, wählt dann für $\alpha$ und $\delta$ die Buchstaben $a$ und $b$, so erhält man den Satz:

Sind $a, b, z$ Functionen von $x$, und finden zwischen ihnen die drei Gleichungen

$$
\left\{\begin{aligned}
N z & =a \sqrt{1-\varkappa_{1}^{2} b^{2}}+i b \sqrt{1-a^{2}} \sqrt{1-b^{2}} \sqrt{1-\varkappa^{2} a^{2}} \\
N_{\sqrt{1-z^{2}}} & =\sqrt{1-a^{2}} \sqrt{1-b^{2}}-i a b \sqrt{1-\varkappa^{2} a^{2}} \sqrt{1-\varkappa_{1}^{2} b^{2}} \\
N \sqrt{1-\varkappa^{2} z^{2}} & =\sqrt{1-b^{2}} \sqrt{1-\varkappa^{2}} \overline{a^{2}} \sqrt{1-\varkappa_{1}^{2} b^{2}}-i \varkappa^{2} a b \sqrt{1-a^{2}}
\end{aligned}\right.
$$

Statt, wo zur Abkürzung

$$
N=1-b^{2}+x^{2} a^{2} b^{2}
$$

gesetzt ist, so wird

$$
\frac{z^{\prime}}{\sqrt{1-z^{2}} \sqrt{1-x^{2} z^{2}}}=\frac{a^{\prime}}{\sqrt{1-a^{2}} \sqrt{1-x^{2} a^{2}}}+\frac{i b^{\prime}}{\sqrt{1-b^{2}} \sqrt{1-x_{1}^{2} b^{2}}} .
$$

Hier bedeuten $a^{\prime}, b^{\prime}, z^{\prime}$ die Differentialcoefficienten von $a, b, z$ nach $x$, ferner $x_{1}$ eine $\sqrt{1-x^{2}}$. Die Zeichen der sechs Quadratwurzeln können beliebig genommen werden, wenn sie nur den Gleichungen (1.) genügen. Natürlich 26 * 
müssen sie in den drei Gleichungen (1.) und in der Differentialgleichung dieselben Werthe haben.

Um sich von den verschiedenen Methoden, durch welche diese Gleichungen hergeleitet werden, unabhängig zu machen, kann man dieselben verificiren. Dividirt man die erste Gleichung in (1.) durch $N$, differentiirt sie dann nach $x$, und dividirt durch das Product der beiden anderen, so findet man ohne grofse Rechnung die Differentialgleichung. Eben so leicht zeigt sich, dafs wenn die rechte Seite der ersten Gleichung in (1.) gleich $\mathrm{Nz}$ gesetzt ist, das Quadrat der rechten Seite der zweiten und der dritten resp. $N^{2}\left(1-z^{2}\right)$ und $N^{2}\left(1-\varkappa^{2} z^{2}\right)$ ist.

Aus der Gleichheit zweier Functionen folgt auch die ihrer bestimmten Integrale zwischen beliebigen reellen Grenzen $g$ und h. Daher läfst sich die Differentialgleichung auch durch

$$
\int_{g}^{h} \frac{z^{\prime} d x}{\sqrt{1-z^{2}} \sqrt{1-x^{2} z^{2}}}=\int_{g}^{\cdot h} \frac{a^{\prime} d x}{\sqrt{1-a^{2}} \sqrt{1-x^{2} a^{2}}}+i \int_{g}^{h} \frac{b^{\prime} d x}{\sqrt{1-b^{2}} \sqrt{1-x_{1}^{2} b^{2}}}
$$

ersetzen.

\section{\$. 2 .}

Für einen grofsen Theil der folgenden Rechnungen sind die Gleichungen (1.) nicht bequem; man kann für sie das System von drei anderen, nämlich

$$
\begin{aligned}
\sqrt{1-b^{2}} \sqrt{1-z^{2}} & =\sqrt{1-a^{2}}-i a b \sqrt{1-\varkappa^{2} z^{2}} \\
z \sqrt{1-b^{2}} \sqrt{1-\varkappa^{2} a^{2}} & =i b \sqrt{1-a^{2}}+a \sqrt{1-\varkappa^{2} z^{2}} \\
z \sqrt{1-\varkappa_{1}^{2} b^{2}} & =a+i b \sqrt{1-a^{2}} \sqrt{1-\varkappa^{2} z^{2}}
\end{aligned}
$$

einführen, die sich leicht aus den ersten herleiten lassen. Beide Systeme sind offenbar gleichbedeutend.

Anmerk. Ebenso wie die Gleichungen (1.) aus den bekannten Formeln für $\sin a m(u+v), \cos a m(u+v), \Delta \operatorname{am}(u+v)$ folgen, so lassen sich 3, 4, und 5 aus den Formeln

$$
\cos \operatorname{am}(u+v)=\operatorname{cosam} u \cos \operatorname{am} v-\sin \operatorname{am} u \sin \operatorname{am} v \Delta \operatorname{am}(u+v)
$$

ferner aus

$\sin \operatorname{am}(u+v) \Delta \operatorname{am} v=\sin$ am $u \cos a m v+\sin \operatorname{am} v \cos a m u \Delta a m(u+v)$

und der Gleichung herleiten, welche aus letzterer nach Vertauschung von $u$ mit $v$ entsteht. 
\$. 3.

\section{Das Integral}

$$
\boldsymbol{J}=\int_{g}^{h} \frac{z^{\prime} d x}{\sqrt{1-z^{2}} \sqrt{1-x^{2} z^{2}}},
$$

über dessen Zerlegung in dieser ersten Abtheilung gehandelt wird, ist ein fest bestimmtes, wenn au/ser den Grenzen $g$ und $h$ auch die Function unter dem Integrale gegeben ist. $\mathrm{Zu}$ dem Letzteren reicht es aber nicht hin, wenn aufser dem Modulus $*$ noch der Gang von $\approx$, d. h. die Art bekannt ist, auf welche $z$ von $x$ abhängt: es mufs auch noch das Zeichen der Quadratuurzeln, oder wenigstens das Zeichen ihres Productes gegeben sein.

Wir nehmen an, da/s z sich stätig ändert; alsdann kann das Zeichen der Quadratwurzeln in $\boldsymbol{J}$ so bestimmt sein, da/s dieselben sich gleichfalls stätig ändern, oder so, dafs sie zuweilen endliche Sprünge machen. In dem letzten Falle kann man $\boldsymbol{J}$ in eine Summe von Integralen derselben Form zerlegen, von denen jedes sich in dem ersten Falle befindet. Wir werden deshalb, ohne die Allgemeinheit zu beschränken, annehmen, da/s die Zieichen der Quadratuurseln in $J$ so bestimmt sind, dafs die Quadratwurzeln sich continuirlich ändern.

Die Bestimmung, welche hier getroffen wurde, ist nicht gleichbedeutend mit der, dafs der reelle Theil der Quadratwurzeln ein bestimmtes Zeichen behalten soll. Ganz abgesehen davon, dafs bei der letzteren Festsetzung das Zeichen unbestimmt bleibt, wenn z. B. in $\sqrt{1-z^{2}}$ die Gröfse $z$ reell und gröfser als 1 wird, so würde auch bei derselben die Continuität unterbrochen werden, wenn $z$ von einem Werthe mit positivem jmaginären Theile zu einem anderen mit negativem durch einen reellen Werth übergeht, der gröfser als 1 ist. Die Behandlung eines Integrals $\boldsymbol{J}$ bei solcher Bestimmung würde daher eine Zerlegung nöthig machen, wie sie oben angedeutet ist.

Kennt man das Zeichen der Wurzeln für irgend einen Werth von $x$, so lä/st es sich nach unseren Festsetzungen für jeden anderen Werth von $x$ ableilen, wenn $z$ nicht dazwischen durch \pm 1 oder $\pm \frac{1}{x}$ gegangen ist. Wir zerlegen deshalb unser Integral noch weiter in eine Summe solcher, in denen $z$ entweder. überhaupt nicht, oder nur an der unteren, oder nur an der oberen, oder an beiden Grenzen den Werth $\pm 1, \pm \frac{1}{x},-$ wir fügen noch aus Gründen, die später klar werden, 0 hinzu - annimmt. Jedes von diesen 
Integralen ist bestimmt, wenn man aufser den Constanten $g, h, \varkappa$ und dem Gange von $z$ noch das Zeichen der Wurzeln für irgend einen Werth von $x$, z. B. für den Anfangswerth $g$ kennt. Von nun an soll $\boldsymbol{J}$ ein solches Integral vorstellen.

\section{S. 4.}

Was oben über Continuität oder Discontinuität der Wurzeln gesagt wurde, zeigt sich leicht durch Anwendung der bekannten Formeln, welche die Ausziehung von Quadratwurzeln aus imaginären Ausdrücken betreffen. Man kann sich zu diesem $Z$ wecke auch der nachfolgenden Transformation bedienen, die sich auf die Form $\sqrt{c^{2}-(m+n i)^{2}}$ bezieht, wenn $c, m, n$ reelle Zahlen bezeichnen, und $c$ positiv ist.

Man kann aus diesen Grölsen durch die Gleichungen

$$
\begin{aligned}
& c=\varrho \sin \theta \\
& n=\sqrt{\varrho^{2}-m^{2}} \cdot \cos \theta
\end{aligned}
$$

die elliptischen Coordinaten $\rho$ und $\theta$ finden, und zwar $\operatorname{sind} \varrho, \sin \theta$ und $\cos \theta$ vollkommen bestimmt, wenn $\varrho$ und $\sqrt{\varrho^{2}-m^{2}}$ reell und positiv und auch $\theta$ reell genommen wird. Denn die Gleichung

hat, da

$$
\frac{c^{2}}{\varrho^{2}}+\frac{n^{2}}{\varrho^{2}-m^{2}}=1
$$

$$
\varrho^{2}\left(\varrho^{2}-m^{2}\right)-n^{2} \varrho^{2}-c^{2}\left(\rho^{2}-m^{2}\right)
$$

für $\varrho^{2}$ gleich $\infty, m^{2}$ und 0 resp. positiv, negativ und positiv wird, nur einen positiven Werth für $\varrho^{2}$, der gröfser als $m^{2}$ ist. Man findet dann

$$
\sqrt{c^{2}-(m+n i)^{2}}= \pm\left(\sqrt{\rho^{2}-m^{2}}-i m \cos \theta\right) \text {. }
$$

Um diese Formel auf $\sqrt{1-z^{2}}$ und $\sqrt{1-\varkappa^{2} z^{2}}$ anzuwenden, hat $\operatorname{man} c=1$ und $z$ oder resp. $x z$ gleich $m+n i$ zu setzen.

\section{S. 5.}

Man zerlege nun $\boldsymbol{J}$ nach der Formel (2.) in die Summe zweier Integrale und versuche $a$ und $b$ so zu bestimmen, da/s sie reell und kleiner (nicht gröfser) als 1 werden. Eine Zerlegung von $J$ in die Summe zweier elliptischen Integrale kann natürlich auf unendlich viele verschiedene Arten Statt finden; aber die Bedingungen, welche wir so eben hinzufügten, bestimmen vollkommen eine Zerlegung. 
Um dieselbe zu bewerkstelligen, ist es nothwendig, erstens die Zahlwerthe von $a$ und $b$, dann auch die Zeichen von $a, b, \sqrt{1-a^{2}}$ etc. oder wenigstens gewisser Combinationen derselben anzugeben. Zu dieser Bestimmung reichen die sechs Gleichungen aus, die man aus 3,4 und 5 erhält, indem man ihre reellen und imaginären Theile für sich gleich setzt. Damit dies bequemer geschehen könne, bezeichnen wir die zu $z, x, x_{1}$ conjugirten Zahlen ${ }^{*}$ ) mit $\zeta, \gamma, \gamma_{1}$, ferner die zu $\sqrt{1-z^{2}}, \sqrt{1-\varkappa^{2} z^{2}}, \sqrt{1-\varkappa^{2} a^{2}}$, etc. conjugirten Zahlen mit $\sqrt{1-\zeta^{2}}, \sqrt{1-\gamma^{2} \zeta^{2}}, \sqrt{1-\gamma^{2} a^{2}}$, etc. und führen endlich folgende häufig vorkommenden Verbindungen ein:

$$
\begin{aligned}
p & =\sqrt{1-\varkappa^{2} z^{2}}+\sqrt{1-\gamma^{2} \zeta^{2}} \\
q i & =\sqrt{1-z^{2}}-\sqrt{1-\zeta^{2}} \\
r & =\sqrt{1-z^{2}} \sqrt{1-\gamma^{2} \zeta^{2}}+\sqrt{1-\zeta^{2}} \sqrt{1-\varkappa^{2} z^{2}}
\end{aligned}
$$

so dafs also $p, q, r$ bekannte reelle Gröfsen sind.

Da der besondere Fall, dafs $\varkappa$ reell also $\gamma=\varkappa$ wird, von Interesse ist und eine einfachere Behandlung gestattet, so wollen wir uns zunächst mit ihm beschäftigen.

\section{Besonderer Fall: $*$ ist reell.}

\$. 6 .

Um den Werth von $a$ zu finden, setzen wir die imaginären Theile von (3.) gleich, ebenso die reellen und erhalten

$$
\text { (a.) } \quad \begin{aligned}
a b p & =-q \sqrt{1-b^{2}} \\
a p & =(z+\zeta) \sqrt{1-b^{2}} \sqrt{1-\varkappa^{2} a^{2}} .
\end{aligned}
$$

Statt der letzten Gleichung benutzen wir die folgende:

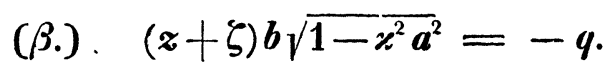

Aus ( $\alpha$. ) und ( $\beta$.) eliminirt man $b$, indem man beide zum Quadrat erhebt, und dann eine von der andern subtrahirt. Dadurch ergiebt sich der gesuchte Werth

$$
\text { (6.) } \quad a^{2}=\frac{(z+\zeta)^{2}-q^{2}}{x^{2}(z+\zeta)^{2}+p^{2}} \text {. }
$$

Aus (5.) läfst sich $b$ finden, indem diese Gleichung wieder in zwei zerfällt wird, von denen die eine

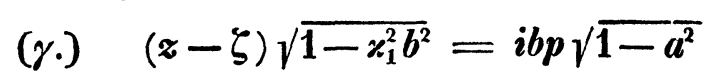

*) Bekanntlich heifst $a-b i$ die zu $a+b i$ conjugirte Zahl. 
ist, die andere gleichfalls linear nach $\sqrt{1-a^{2}}$. Eliminirt man diese Gröfse, so findet man

$$
\sqrt{1-\varkappa_{1}^{2} b^{2}}=\frac{a p}{z \sqrt{1-x^{2} \zeta^{2}}+\zeta \sqrt{1-x^{2} z^{2}}}
$$

Anmerk. Im $45^{\text {sten }}$ Bande dieses Journals giebt Richelot p. 227 Formeln für $a$ und $\sqrt{1-\varkappa_{1}^{2} b^{2}}$, die in anderer Gestalt auftreten. Wenn wir unsere Bezeichnung beibehalten, so sind seine Ausdrücke

$$
\begin{aligned}
\left(6^{*} .\right) \quad \boldsymbol{a} & = \pm \frac{\sqrt{1+z} \sqrt{1+\zeta}-\sqrt{1-z} \sqrt{1-\zeta}}{\sqrt{1+x z} \sqrt{1+x \zeta}+\sqrt{1-x z} \sqrt{1-x \zeta}} \\
\left(7^{*} .\right) \quad \sqrt{1-x_{1}^{2} b^{2}} & = \pm \frac{\sqrt{1+x z} \sqrt{1+x \zeta}+\sqrt{1-x z} \sqrt{1-x \zeta}}{\sqrt{1+z} \cdot \sqrt{1+\zeta}+\sqrt{1-z} \sqrt{1-\zeta}} .
\end{aligned}
$$

Die Uebereinstimmung dieser Werthe mit den obigen ergiebt sich leicht. Multiplicirt man nämlich Zähler und Nenner in (6.) mit 2, so sind dieselben unmittelbar die Quadrate der Zähler und Nenner von $\left(6^{*}\right.$.). Die Gleichheit der andern Formeln zeigt sich, wenn man Zähler und Nenner in (7.) mit $\sqrt{1-\varkappa^{2} \approx^{2}}-\sqrt{1-\varkappa^{2} \zeta^{2}}$ multiplicirt, und die Identität

$$
\left(\sqrt{1+x z} \sqrt{1+x^{\zeta} \zeta}-\sqrt{1-x^{2}} \sqrt{1-x^{\zeta} \zeta}\right)^{2}=2\left(1+x^{2} z \zeta-\sqrt{1-x^{2} z^{2}} \sqrt{1-x^{2} \zeta^{2}}\right)
$$

benutzt.

\section{\$. 7.}

Die gefundenen Werthe von a und b sind offenbar reell, man sieht ferner leicht ein, dafs sie kleiner als 1 sind. Zunächst folgt nämlich aus $(\alpha$.$) , dafs \sqrt{1-b^{2}}$ reell, d. h. dafs $b<1$ ist. Ferner sieht man aus $(\gamma$.$) ,$ dafs $\sqrt{1-a^{2}}$ reell, d. h. $a<1$ ist.

Ferner sind $a^{2}$ und $b^{2}$ continuirliche Functionen von $x$. Es könnte $a^{2}$ nur discontinuirlich werden, wenn der Nenner in (6.) verschwindet, d. h. wenn zugleich $z+\zeta$ und $p$ gleich 0 sind. Es ist aber $z+\zeta=0$, wenn $z$ rein imaginär $=n i$ ist; dann wird $p=2 \sqrt{1+x^{2} n^{2}}$, also gewifs nicht 0 . Aus ( $\beta$.) folgt die Continuität von $b^{2}$; selbst wenn $z+\zeta=0$ ist, mufs $b^{2}$ continuirlich bleiben, indem selbst in diesem Falle, d. b. für ein rein imaginäres $z$ der Ausdruck $\frac{q i}{z+\zeta}=\frac{\sqrt{1-z^{2}}-\sqrt{1-\zeta^{2}}}{z+\zeta}=\frac{\zeta+z}{\sqrt{1-z^{2}}+\sqrt{1-\zeta^{2}}}$ continuirlich bleibt, und $\sqrt{1-\varkappa^{2} a^{2}}$ nie verschwindet.

Einfache Combinationen der Gleichungen des vorigen Paragraphen lehren, für welche $z$ die Grö/sen $a^{2}$ und $b^{2}$ ihren grö/sten und kleinsten Werth 
1 und 0 erreicleen. Es wird

$$
\begin{aligned}
& a^{2}=0 ; \text { wenn } z \text { rein imaginär ist, } \\
& a^{2}=1 ; \text { wenn } z \text { reell ist, und dabei } z \text { wischen } 1 \text { und } \frac{1}{x} \text { liegt, } \\
& b^{2}=0 ; \text { wenn } z \text { reell und }<1, \\
& b^{2}=1 ; \text { wenn } z \text { reell und }>\frac{1}{x} .
\end{aligned}
$$

Die Grenzfälle sind hier überall eingeschlossen; z. B. wird a noch gleich 0 für $:=0$.

Stellt man die imaginären Gröfsen auf bekannte Art geometrisch dar, nimmt die Achse der $\boldsymbol{X}$ als Achse des Reellen (Fig. 1.), die der $\boldsymbol{Y}$ als Achse des rein Imaginären, sind ferner $\boldsymbol{A} \boldsymbol{X}$ und $\boldsymbol{A} \boldsymbol{Y}$ die positiven Seiten, so kann man die Werthe 1 und $\frac{1}{x}$ durch $\boldsymbol{M}$ und $\boldsymbol{N}$ repräsentiren (indem man $\varkappa$ die positive Wursel aus $\varkappa^{2}$ nennt), -1 und $-\frac{1}{x}$ durch $M^{\prime}$ und $N^{\prime}$. Es wird dann $a=0$ für alle Puncte, die in $Y^{\prime} Y$ liegen; $a^{2}=1$ für die Linien $M N$ und $M^{\prime} N^{\prime}$; es wird $b=0$ für das Stück $M^{\prime} M$; $b^{2}=1$ für $\boldsymbol{N} \boldsymbol{X}$ und $\boldsymbol{N}^{\prime} \boldsymbol{X}^{\prime}$.

Fig. 1.

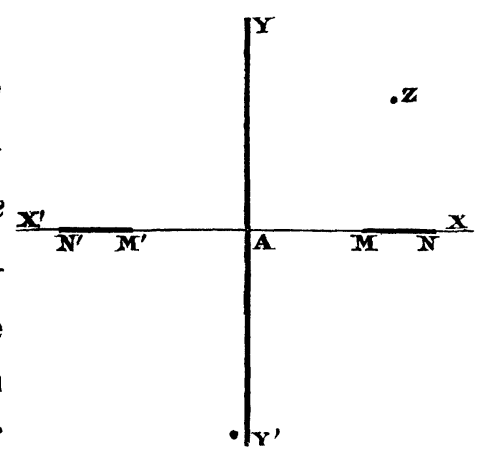

§. 8 .

Indem wir nun zur Zeichenbestimmung übergehen, bemerken wir vorläufig, da/s es nur nöthiy ist, die Vorseichen der beiden Ausdrücke

$$
\begin{aligned}
& a \sqrt{1-a^{2}} \sqrt{1-\varkappa^{2} a^{2}}=A \\
& b \sqrt{1-b^{2}} \sqrt{1-\varkappa_{1}^{2} b^{2}}=B
\end{aligned}
$$

zu kennen; die Zeichen der sechs Factoren von $\boldsymbol{A}$ und $\boldsymbol{B}$ bleiben der Natur der Sache nach unbestimmt. Nachdem $\$$. 7. gezeigt wurde, dafs $a^{2}$ und $b^{2}$ continuirliche Functionen von $x$ sind, wird sich zunächst beweisen lassen, dafs auch $A$ und $B$ continuirlich sind, ihr Zeichen also nur für $a=0$, $b=0, a^{2}=1, b^{2}=1$ ändern können.

Zur Abkürzung soll $[Z]$ entweder $+1,-1$ oder Null vorstellen, je nachdem $\boldsymbol{Z}$ positiv, negativ oder Null ist.

Multiplicirt man die reellen Theile der ersten und zweiten Gleichung von (1.) mit einander, und dividirt durch den reellen Theil der dritten, so findet man

$$
\frac{2 a \sqrt{1-a^{2}}}{\sqrt{1-x^{2} a^{2}}}=\frac{N(z+\zeta)\left(\sqrt{1-z^{2}}+\sqrt{1-\zeta^{2}}\right)}{p}
$$

Journal f. d. M. Bd. LIIl. Heft 3. 
und auf ähnliche Art:

$$
2 b \sqrt{1-b^{2}} \sqrt{1-\varkappa_{1}^{2} b^{2}}\left(1-\varkappa^{2} a^{2}\right)=N \frac{z-\zeta}{i} \frac{p}{\sqrt{1-z^{2}}+\sqrt{1-\zeta^{2}}} .
$$

Da $p$ nur verschwindet, wenn $\sqrt{1-\varkappa^{2} z^{2}}$ rein imaginär, d. h. $\approx$ reell und $>\frac{1}{x}$ ist, und in diesem Falle auch $\sqrt{1-z^{2}}+\sqrt{1-\zeta^{2}}=0$, der Quotient

aber gleich

$$
\frac{\sqrt{1-z^{2}}+\sqrt{1-\zeta^{2}}}{\sqrt{1-x^{2} z^{2}}+\sqrt{1-x^{2} \zeta^{2}}}
$$

$$
\frac{1}{x^{2}} \cdot \frac{\sqrt{1-z^{2}}-\sqrt{1-\zeta^{2}}}{\sqrt{1-x^{2} z^{2}}-\sqrt{1-x^{2} \zeta^{2}}}
$$

ist, und gewifs nicht verschwindet, so ist die rechte also auch die linke Seite der ersten von den vorstehenden Gleichungen continuirlich. Auf ähnliche Art folgt die Continuität beider Seiten der zweiten Gleichung. Da ferner $1-\varkappa^{2} a^{2}$ nie verschwindet, so sind $\boldsymbol{A}$ und $\boldsymbol{B}$ continuirlich, und ihre Zeichen sind durch die Gleichungen

$$
\begin{aligned}
& {[\boldsymbol{A}]=\left[(\boldsymbol{z}+\zeta) \frac{\sqrt{1-z^{2}}+\sqrt{1-\zeta^{2}}}{\sqrt{1-x^{2} z^{2}}+\sqrt{1-x^{2} \zeta^{2}}}\right]} \\
& {[\boldsymbol{B}]=\left[\frac{z-\zeta}{i} \frac{\sqrt{1-x^{2} z^{2}}+\sqrt{1-x^{2} \zeta^{2}}}{\sqrt{1-z^{2}}+\sqrt{1-\zeta^{2}}}\right]}
\end{aligned}
$$

gegeben. Will man also den Zeichen von $\boldsymbol{A}$ für den ganzen Lauf von $z$ folgen, so wird man zunächst aus den Anfangswerthen von $z$ das Anfangszeichen von $\boldsymbol{A}$ berechnen; dasselbe Zeichen von $\boldsymbol{A}$ bleibt sicher so lange, bis $a$ gleich 0 oder \pm 1 wird, d. h. (\$. 7.) bis $z$ rein imaginär oder reell, gröfser als 1 und kleiner als $\frac{1}{x}$ ist.- Erst dann hat man eine neue Rechnung für $[\boldsymbol{A}]$ anzustellen. Ähnlich ist es mit $[\boldsymbol{B}]$.

Diese neue Rechnung, oder vielmehr die jedesmalige Wiederholung derselben Rechnung an den kritischen Stellen kann man jedoch ersparen. Wird $\mathbf{z}$ wie oben gleich $m+n i$ gesetzt, so sind jene kritischen Stellen die, an denen $\boldsymbol{m}$ oder $\boldsymbol{n}$ verschwindet; je nachdem die verschwindende Gröfse ihr Zeichen behält oder wechselt, wird auch $\boldsymbol{A}$ oder $\boldsymbol{B}$ sein Zeichen behalten oder wechseln.

Um dies zu zeigen, kann man auf die Formeln des $\$$. 4. zurückgehen. Verschwindet zunächst $m$ an einer Stelle, so wird $\sqrt{1-z^{2}}$ eine reelle Gröfse, die dasselbe Zeichen hat wie der reelle Theil dieser Wurzel für das unmittelbar vorhergehende oder folgende $z$. Es folgt ferner daraus, dafs der imaginäre Theil der Wurzel sein Zeichen behält oder wechselt, je nachdem $m$ es behält oder wechselt. Das Gleiche gilt von $\sqrt{1-\varkappa^{2} z^{2}}$. 
Verschwindet $\boldsymbol{u}$ an einer Stelle, so sind bei Betrachtung von $\sqrt{1-z^{2}}$ zwei Fälle zu unterscheiden. Ist erstens $m<1$, so wird der reelle Theil der Wurzel sein Zeichen behalten, der imäginäre es mit $n$ zugleich wechseln. Ist zweitens $m>1$, so wird der imaginäre Theil sein Zeichen behalten, der reelle es mit $n$ wechseln. Hieraus folgt ferner für $\sqrt{1-\varkappa^{2} z^{2}}$, dafs wenn $m<\frac{1}{x}$ ist, der reelle Theil sein Zeichen behält, der imaginäre es mit $n$ wechselt; wenn $m>\frac{1}{x}$, so behält es der imaginäre, während der reelle es mit $n$ wechselt.

Unter Anfangswerth von $[\boldsymbol{A}]$ und $[\boldsymbol{B}]$ verstehen wir den, welcher zuerst nicht Null ist.

Es läfst sich nun leicht erkennen, ob an einer kritischen Stelle $\boldsymbol{A}$ und $\boldsymbol{B} \mathrm{ihr}$ Zeichen ändern. Wird $a=0 \mathrm{~d}$. h. geht $z$ durch das rein Imaginäre, so wird $z+\zeta=2 m$ sein Zeichen mit $m$ wechseln. Die übrigen Gröfsen in [A] behalten ihr Zeichen; also wechselt $A$ sein Zeichen mit $m$. Wird $a^{2}=1$ so geht $z$ durch den reellen Werth $m$, der gröfser als 1 und kleiner als $\frac{1}{x}$ ist. Also behält $z+\zeta=2 m$ sein Zeichen, ebenso $\sqrt{1-\varkappa^{2} z^{2}}+\sqrt{1-\varkappa^{2} \zeta^{2}}$, während $\sqrt{1-z^{2}}+\sqrt{1-\zeta^{2}}$ es mit $n$ wechselt; also wechselt $A$ sein Zeichen mit $n$. Ist $b=0$ so erhält $z$ einen Werth $m$, der kleiner als 1 ist; $\frac{z-\zeta}{i}$ ist $2 n$ und wechselt daher sein Zeichen mit $n$. Es wechselt also $B$ sein Zeichen mit $n$. Wird $b^{2}=1$, so ist $z$ und $\varkappa z$ gröfser als 1 ; es wechselt also $\boldsymbol{B}$ sein Zeichen mit $n$. Wir haben also folgende Resultate: Wechselt der reelle Theil von $\approx$ sein Zeichen, oder wechselt der imaginäre Theil von $z$ sein Zeichen, während hierbei der reelle zwischen 1 und $\frac{1}{x}$ liegt, so wechselt es auch, und nur in diesen Fällen, $\boldsymbol{A}$; es ist dann der Werth von $\boldsymbol{a}^{2}$ resp. 0 oder 1. Wechselt der imaginäre Theil von $z$ sein Zeichen, und ist der reelle dabei $<1$ oder $>\frac{1}{x}$, und nur dann wechselt $B$ sein Zeichen; es ist dann $b^{2}$ resp. 0 oder 1. Geometrisch genommen wechselt $\boldsymbol{A}$ sein Zeichen, wenn die Curve $z$ die stärker gezogenen, $\boldsymbol{B}$ wenn sie die schwächeren Theile der Achsen in Fig. 1. durchschneidet, und zwar bedeuten der Reihe nach die obigen vier Fälle, dafs die Curve $z$ die Achse $\boldsymbol{Y}^{\prime} \dot{\boldsymbol{Y}}$, das Stück $M N$ oder $M^{\prime} N^{\prime}$, drittens $M^{\prime} M$ und viertens $\boldsymbol{N} \boldsymbol{X}$ oder $\boldsymbol{N}^{\prime} \boldsymbol{X}^{\prime}$ durchschneidet.

Fig. 1.

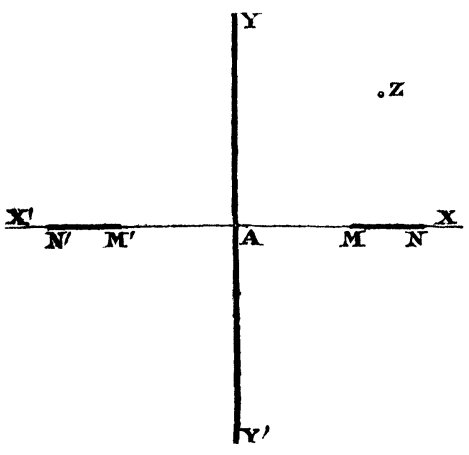


Solange $[\boldsymbol{A}]=0$ oder $[\boldsymbol{B}]=0$ behält $\boldsymbol{a}$ und resp. $\boldsymbol{b}$ denselben Werth; z bleibt dann auf einer der Achsen.

Wir folgen nun dem Laufe von $z$ und bezeichnen der Reihe nach die Werthe von $a$ und $b$, bei denen eine solche Zeichenänderung Statt findet, oder vielmehr ihre Zahlwerthe mit $\alpha_{1}, \alpha_{2}, \ldots \alpha_{m}$, resp. mit $\beta_{1}, \beta_{2}, \ldots \beta_{n}$. Die $\alpha$ und $\beta$ sind also 0 und +1 . Es seien ferner $\alpha_{1}, \mathfrak{A}, \beta_{0}, \mathfrak{B}$ die Zablwerthe von den Anfangs - und Endwerthen von $a$ und $b$, die also aus (6.) und (7.) berechnet werden, indem man in diese Formeln das z einsetzt, welches $x=g$ und $x=h$ entspricht. Die Anfangswerthe von $[\boldsymbol{A}]$ und $[\boldsymbol{B}]$ seien endlich $\left[\boldsymbol{A}_{0}\right]$ und $\left[\boldsymbol{B}_{0}\right]$.

\section{S. 9.}

Nach diesen Vorbereitungen hat die Zerlegung von $\boldsymbol{J}$, d. h. also von

$$
\int \frac{d z}{\sqrt{1-z^{2}} \sqrt{1-x^{2} z^{2}}}
$$

wo z auf gegebenem Wege von einem gegebnen Anfangswerthe zu einem gleichfalls gegebnen Endwerthe wächst, keine Schwierigkeiten. In die Formel (2.) hat man für $a$ und $b$ die Werthe zu setzen, die ihnen nach $\$$. 6. zukommen. Sind nun $\alpha$ und $\beta$ die Zahlenwerthe von $a$ und $b$, und verstehen wir unter $\sqrt{1-\alpha^{2}}, \sqrt{1-\varkappa^{2} \alpha^{2}}, \sqrt{1-\beta^{2}}, \sqrt{1-\varkappa_{1}^{2} \beta^{2}}$ die positiven Wurzeln, so wird für das Glied

in (2.) jetzt

$$
\frac{a^{\prime}}{\sqrt{1-a^{2}} \sqrt{1-x^{2} a^{2}}}, \quad \frac{b^{\prime}}{\sqrt{1-b^{2}} \sqrt{1-x_{1}^{2} b^{2}}}
$$

$$
[\boldsymbol{A}] \frac{\alpha^{\prime}}{\sqrt{1-\alpha^{2}} \sqrt{1-x^{2} \alpha^{2}}}, \quad[\boldsymbol{B}] \frac{\beta^{\prime}}{\sqrt{1-\beta^{2}} \sqrt{1-x_{1}^{2} \beta^{2}}}
$$

selbst an den Stellen zu setzen sein, an welchen $[\boldsymbol{A}]$ und $[\boldsymbol{B}]$ Null sind (weil für diese $a$ und $b$ constant sind). Zerlegen wir die beiden Integrale auf der rechten Seite in eine Summe solcher, in denen $\boldsymbol{A}$ und $\boldsymbol{B}$ ihre Zeichen nicht ändern, so wird schliefslich

$$
\begin{aligned}
J & =\left[\boldsymbol{A}_{0}\right]\left\{\int_{\alpha_{0}}^{\alpha_{1}} \frac{d \alpha}{\sqrt{1-\alpha^{2}} \sqrt{1-x^{2} \alpha^{2}}}-\int_{\alpha_{1}}^{\alpha_{2}}+\cdots+(-1)^{m} \int_{\alpha_{m}}^{\mathfrak{2}}\right\} \\
& +i\left[\boldsymbol{B}_{0}\right]\left\{\int_{\beta_{n}}^{\beta_{1}} \frac{d \beta}{\sqrt{1-\beta^{2}} \sqrt{1-x_{1}^{2} \beta^{2}}}-\int_{\beta_{1}}^{\beta_{2}}+\cdots+(-1)^{n} \int_{\beta_{n}}^{\mathfrak{B}_{1}}\right\} .
\end{aligned}
$$

Die Function, welche unter dem Integrale steht, ist in der ersten Zeile 
$\frac{d \alpha}{\sqrt{1-\alpha^{2}} \sqrt{1-x^{2} \alpha^{2}}}$, in der zweiten $\frac{d \beta}{\sqrt{1-\beta^{2}} \sqrt{1-x_{1}^{2} \beta^{2}}}$, und der Werth eines jeden Integrales, mit Ausnahme des ersten und letzten jeder Zeile, in der ersten 0 oder $\boldsymbol{K}$, in der zweiten 0 oder $\boldsymbol{K}^{\prime}$. Man wird also die Zerlegung leicht vornehmen können, es mag $z$ analytisch gegeben oder als Curve gezeichnet sein.

Als Beispiel wählen wir einen besonders wichtigen Fall, nämlich den, wo $z$ in $\boldsymbol{J}$ von 0 zu einem gegebnen Werthe $\boldsymbol{Z}$ geht, ohne jemals rein reell oder imaginär zu werden. Für $z=0$ mögen $\sqrt{1-z^{2}}$ und $\sqrt{1-\varkappa^{2} z^{2}}$ gleich +1 sein. Es ist also $z$ eine beliebige Curve oder Gerade, die $\boldsymbol{A}$ mit $\mathbf{Z}$ verbindet, ohne die Achsen zu schneiden. Es ist dann $\left[\boldsymbol{A}_{0}\right]$ offenbar +1 , wenn $\boldsymbol{Z}$ im ersten oder vierten Quadranten liegt, sonst $-\mathbf{1} ;\left[\boldsymbol{B}_{0}\right]$ gleich $+\mathbf{1}$, für ein $\boldsymbol{Z}$ im ersten oder zweiten Quadranten, sonst -1 . Ferner ist $\alpha_{0}=\beta_{0}=0$. $\mathfrak{A}$ und $\mathfrak{B}$ werden nach (6.) und (7.) für $\mathfrak{z}=\boldsymbol{Z}$ berechnet, und dann ist $\boldsymbol{J}$ die Summe der zwei Integrale

$$
\left[\boldsymbol{A}_{0}\right] \int_{0}^{\mathfrak{A}} \frac{d \alpha}{\sqrt{1-\alpha^{2}} \sqrt{1-x^{2} \alpha^{2}}}+i\left[\boldsymbol{B}_{0}\right] \int_{0}^{\mathscr{B}} \frac{d \beta}{\sqrt{1-\beta^{2}} \sqrt{1-x_{1}^{2} \beta^{2}}} \cdot
$$

Man wird übrigens leicht bemerken, dafs durch (8.) die Eigenschaft der Periodicität für die elliptischen Integrale streng bewiesen ist.

\section{Allgemeiner Fall: $\varkappa$ ist imaginär.}

\section{\$. 10.}

Die Untersuchungen der vorigen Paragraphen lassen sich leicht auf den Fall ausdehnen, dafs $\varkappa$ eine imaginäre Zahl ist; der Fall, dafs $\varkappa$ rein imaginär wird, der sich auf den eines reellen $\varkappa$ ohne Mühe zurückführen läfst, soll hier ausgeschlossen werden. Den reellen Theil von $\varkappa$ denken wir uns positiv, $\varkappa \gamma$ wie früher (\$. 1.) kleiner als 1 .

Entsprechend dem \$. 6. werden hier die Formeln für $a$ und $b$ entwickelt. Dazu bedienen wir uns der Gleichung

$$
\text { (ঠ.) } \quad a b p=-q \sqrt{1-b^{2}}
$$

und einer zweiten, die aus Gleichsetzung der reellen Theile von (3.) entsteht; diese geht mit Hülfe von $(\delta$.$) in$

$$
\text { (E.) } \quad \boldsymbol{a b r}=-q \sqrt{1-a^{2}}
$$

über. Die Elimination von $a$ oder $b$ aus $(\delta$.$) und \left(\varepsilon_{0}\right)$ giebt

(9.) $\quad p^{2} a^{4}-a^{2}\left(p^{2}-q^{2}-r^{2}\right)-q^{2}=0$

(10.) $r^{2} b^{4}-b^{2}\left(r^{2}-p^{2}-q^{2}\right)-q^{2}=0$, 
so dafs $a$ und $b$ aus bekannten Stücken $p, q$ und $r$ gefunden werden können. Es ergeben sich nämlich für $a^{2}$ und $b^{2}$ die zwei Werthe

$$
\begin{aligned}
& \text { (11.) } a^{2}=\frac{p^{2}-q^{2}-r^{2} \pm \sqrt{D}}{2 p^{2}} \\
& \text { (12.) } b^{2}=\frac{r^{2}-p^{2}-q^{2} \pm \sqrt{D}}{2 r^{2}}
\end{aligned}
$$

wenn $\boldsymbol{D}$ gleich $\left(q^{2}+r^{2}-p^{2}\right)^{2}+4 p^{2} q^{2}$ gesetzt ist. Es läfst sich übrigens $\boldsymbol{D}$ auch als Product

darstellen.

$$
(r+p+q i)(r+p-q i)(r-p+q i)(r-p-q i)
$$

\section{S. 11.}

Die gefundenen Werthe genügen zwar den ursprünglichen Gleichungen, lösen unsere Aufgabe aber nur dạnn, wenn $a$ und $b$ reell und kleiner als 1 werden. Man wird deshalb in (11.) und (12.) nur die oberen Zeichen nehmen dürfen; die zweite Lösung ist zu verwerfen. In der That wird die linke Seite von (9.) für $a^{2}=1,0,-\infty$ resp. positiv, negativ, positiv, also liegt eine und nur eine Wurzel $a^{2}$ zwischen 0 und 1, die andre ist negativ. Ebenso verhält es sich mit $b^{2}$. Wenn wir von nun an über die Wurzeln $a^{2}$ und $b^{2}$ der Gleichungen (9.) und (10.) handeln, so sind diese allein hierher gehörigen mit den oberen Zeichen gemeint. Zur bequemeren Berechnung derselben kann man nach Anleitung des \$. 4.

$$
\begin{aligned}
& r=\varrho \sin \theta \\
& q=\sqrt{\varrho^{2}-p^{2}} \cos \theta
\end{aligned}
$$

setzen, wodurch man

$$
\text { (13.) }\left\{\begin{array}{l}
a= \pm \cos \theta \\
b= \pm \frac{\sqrt{\varrho^{2}-p^{2}}}{\varrho}
\end{array}\right.
$$

erhält. Die Vorzeichen bleiben natürlich unbestimmt.

Im Folgenden werden $a$ und $b$ für besondere Werthe von $z$ zu betrachten sein; dabei wird man oft bequemer auf (9.) und (10.) als auf die aufgelösten Gleichungen zurückgehen. In einem Fall ist besondere Vorsicht hierbei anzuwenden, nämlich wenn man findet, einem $z$ entspreche $a=0$ oder $\boldsymbol{b}=\mathbf{0}$. In diesem Falle ist nämlich zu untersuchen, ob die zweite Wurzel für $a^{2}$ resp. $b^{2}$ positiv, Null oder negativ ist, und nur in den letzten beiden Fällen ist 0 wirklich die hierher gehörige Wurzel, während sie im ersten Falle nur die Grenze der negativen Wurzel $a^{2}$ oder $b^{2}$ ist. 


\section{\$. 12.}

Die gefundenen Werthe von $a^{2}$ und $b^{2}$ sind continuirlich. Für keinen Werth von $z$ können $p$ und $q$ zugleich verschwinden, da $p=0$ voraussetzt dafs $\varkappa$ reell und gröfser als $1, y=0$ dafs $z$ reell und kleiner als 1 ist. Ist $p=0$, so kann im Allgemeinen nicht $r=0$ sein; denn für $p=0$ wird

$$
\begin{aligned}
r & =-\sqrt{1-\overline{\varkappa^{2} z^{2}}}\left(\sqrt{1-z^{2}}-\sqrt{\left.1-\zeta^{2}\right)}\right. \\
& =-q i \cdot \sqrt{1-\varkappa^{2} s^{2}} .
\end{aligned}
$$

Da aber $q$ mit $p$ nicht zugleich verschwindet, so mufs $x z=1$ sein, ein Fall, der nur am Anfang oder Ende des Laufes von $z$ vorkommen kann.

Es kann $a^{2}$ nach (11.) nur discontinuirlich sein, wenn es für $p=0$ unendlich wird. Aus (9.) ergiebt sich aber für $p=0$

$$
a^{2}=\frac{q^{2}}{q^{2}+r^{2}}
$$

also ein endlicher Werth, der auch positiv ist (da, wie wir eben zeigten, $q$ nicht Null sein kann), also hierher gehört. Auch $b^{2}$ ist continuirlich; eine Discontinuität kann nämlich nur für $r=0$ eintreten, für welchen Fall nach (10.)

$$
b^{2}=\frac{q^{2}}{p^{2}+q^{2}}
$$

wird, also positiv und endlich bleibt, wenn nicht auch $q$ verschwindet, d. h. wenn nicht $z$ gerade gleich \pm 1 wird. Für diesen Fall wird $p$ sicher nicht Null, also der obige Ausdruck für $b^{2}$ gleich Null. Dieser Werth gehört aber wirklich der positiven Wurzel als Grenzfall an, indem für $r=0$ und $\boldsymbol{q}=\mathbf{0}$ aus (9.) unser $a^{2}$ gleich 1 , daher aus $(\delta$.) das hierzugehörige $b=0$ folgt.

Auch hier mu/s man untersuchen, an welchen Stellen $a^{2}$ und $b^{2}$ ihre grö/sten und kleinsten Werthe 1 und 0 annehmen. Damit $a^{2}=0$ sei, mufs nach (9.) zunächst $q=0$ werden. Dann reducirt sich diese Gleichung auf $a^{2}\left(a^{2} p^{2}-p^{2}+r^{2}\right)=0$, so dafs, wenn 0 der Werth der hierher gehörigen Wurzel sein soll, $p^{2}-r^{2}$ negativ sein mufs. Für $q=0$ ist aber $\boldsymbol{r}=\boldsymbol{p} \cdot \sqrt{1-z^{2}}$, und daher mufs $-p^{2} z^{2}$ negativ, d. h. $z$ rein imaginär sein. Die Bedingung $q=0$ wird dann von selbst erfüllt.

Auch wenn $b=0$ sein soll, mufs $q$ verschwinden, aufserdem aber $\frac{r^{2}-p^{2}}{r^{2}}=-\frac{z^{2}}{1-z^{2}}$ negativ sein. Es wird aber $q$ nur Null für ein rein imaginäres $z$, und dann wäre jener Ausdruck positiv; oder wenn $z$ reell und $<1$, und dann wird er wirklich negativ. Es wird also $b=0$ für $z<1$. 
Soll $a^{2}=1$ sein, so mufs nach (9.) nothwendig $r^{2}=0$ sein; $b^{2}$ wird 1 für $p=0$. Wir haben demnach folgende Resultate: Es ist

$$
\begin{aligned}
& a^{2}=0 ; \text { wenn } z \text { rein imaginär wird. Dann ist } q=0 . \\
& b^{2}=0 ; \text { wenn } z<1 . \text { Auch dann ist } q=0 . \\
& a^{2}=1 ; \text { wenn } r=0 . \\
& b^{2}=1 ; \text { wenn } p=0 .
\end{aligned}
$$

Fig. 2.

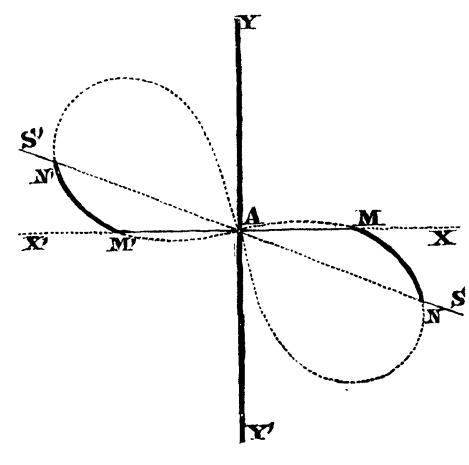

S. 13.

Diese Resultate lassen sich leicht geometrisch deuten (Fig. 2.). Es wird $a^{2}=0$ auf der ganzen Achse des Imaginären $\boldsymbol{Y}^{\prime} \boldsymbol{Y} ; \boldsymbol{b}^{2}=0$ auf dem Stücke $\boldsymbol{M}^{\prime} \boldsymbol{M}$ der reellen Achse, wenn $\boldsymbol{M}$ und $\boldsymbol{M}^{\prime}$ wieder die Repräsentanten von \pm 1 sind. Es mögen $\boldsymbol{N}$ und $\boldsymbol{N}^{\prime}$ wieder $\pm \frac{1}{x}$ vorstellen, dann wird $b^{2}=1$ für die Geraden $N \boldsymbol{S}$ und $\boldsymbol{N}^{\prime} \boldsymbol{S}^{\prime}$, d. h. für die Verlängerung der Geraden $\boldsymbol{N}^{\prime} \boldsymbol{A} \boldsymbol{N}$ in's Unendliche, indem für alle Puncte dieser Geraden $\varkappa z$ reell und $>1$ ist. Es wird endlich $a^{2}=1$ für ein Curvenstück, welches in der Figur $M$ mit $N$ und $M^{\prime}$ mit $N^{\prime}$ verbindel, und welches einer Lemniscate angehört.

Dafs die Bedingung $r=0$ wirklich eine Lemniscate giebt, läfst sich leicht auf folgende Art darthun. Setzt man

$$
s=\sqrt{1-\zeta^{2}} \sqrt{1-\varkappa^{2} z^{2}}-\sqrt{1-z^{2}} \sqrt{1-\gamma^{2} \zeta^{2}}
$$

und bezeichnet die rechtwinkligen Coordinaten eines Punctes $z$, auf die Achsen $\boldsymbol{X}^{\prime} \boldsymbol{X}$ und $\boldsymbol{Y}^{\prime} \boldsymbol{Y}$ bezogen mit $m$ und $n$, so dafs $z=m+n i$ und $\zeta=m-n i$, macht man ferner $\varkappa^{2}=g+h i$, so wird

$$
r s=z^{2}-\zeta^{2}+\gamma^{2} \zeta^{2}-\varkappa^{2} z^{2}+z^{2} \zeta^{2}\left(\varkappa^{2}-\gamma^{2}\right)
$$

also

$$
2 m n(1-y)+h\left(\left(m^{2}+n^{2}\right)^{2}-\left(m^{2}-n^{2}\right)\right)=0 .
$$

Führt man Polarcoordinaten ein

$$
\begin{aligned}
h & =c^{2} \cos 2 \eta \\
1-g & =c^{2} \sin 2 \eta \\
m & =\varrho \cos \theta \\
n & =\varrho \sin \theta,
\end{aligned}
$$

so entsteht die bekannte Polargleichung der Lemniscate

$$
\varrho=\sqrt{\frac{\cos 2(\eta+\theta)}{\cos 2 \eta}} \text {. }
$$


Da der reelle Theil von $\varkappa$ positiv ist, so liegt $\varkappa$ im ersten oder vierten Quadranten. Im ersten Falle ist $h$ positiv, also $\sin 2 \eta$ und $\cos 2 \eta$ positiv. Es ist daher die Länge des gröfsten Radius-Vector $=\frac{1}{\sqrt{\cos 2 \eta}}$. Da ferner $\eta<\frac{\pi}{4}$, so durchschneidet diese Achse den 2ten und 4ten Quadranten und bildet mil der Achse der $\boldsymbol{X}$ im vierten Quadranten einen Winkel, der kleiner als $\frac{\pi}{4}$ ist. Lag $*$ im zweiten Quadranten, so ist jene Länge der halben Achse $\frac{1}{\sqrt{-\cos 2 \eta}}$; sie geht durch den ersten und dritten Quadranten; $\eta$ liegt zwischen $\frac{\pi}{4}$ und $\frac{\pi}{2}$, und die Achse bildet mit der Achse der $\boldsymbol{X}$ im ersten Quadranten den Winkel $\frac{\pi}{2}-\eta$.

Diese Lemniscate genügt aber nicht der Gleichung $\boldsymbol{r}=\mathbf{0}$ sondern $\boldsymbol{r} \boldsymbol{s}=\mathbf{0}$; es bleibt daher zu untersuchen, auf welchem Stïcke $r=0$ ist, indem nur für die vier Puncte $\boldsymbol{M}, \boldsymbol{N}, \boldsymbol{M}^{\prime}, \boldsymbol{N}^{\prime}$ zugleich $r=0$ und $s=0$ wird, weil für $\boldsymbol{r}=\mathbf{0}$ und $s=0$ auch $r \pm s=0$ wäre. Soll $r=0$ sein, so mufs $\sqrt{1-\zeta^{2}} \sqrt{1-\varkappa^{2} z^{2}}$ rein imaginär sein; macht man daher für den Augenblick

$$
\begin{aligned}
\sqrt{1-\zeta^{2}} & =a+b i \\
\sqrt{1-\varkappa^{2} z^{2}} & =e+f i,
\end{aligned}
$$

so folgt daraus $a e-b f=0$, oder $e: f=b: a$. Bezeichnet $m$ eine reelle Zahl, so hat $\sqrt{1-x^{2} z^{2}}$ die Form $\frac{b+a i}{m}$; es ist aber $\sqrt{1-z^{2}}=a-b i$, also

folglich

$$
1-z^{2}=-\left(1-\varkappa^{2} z^{2}\right) n^{2}
$$

$$
z^{2}=\frac{1+m^{2}}{1+x^{2} m^{2}} \text {. }
$$

Selzt man umgekehrt $z^{2}$ gleich diesem Werthe, so wird für jedes reelle $m$ auch $r=0$. Während $m$ von 0 bis $\propto$ wächst, geht offenbar $z^{2}$ von 1 continuirlich $\mathrm{zu} \frac{1}{x^{2}}$, ohne je gleich 0 zu werden: es wird daher $r=0$ für jene Stücke der Lemniscate, die $\boldsymbol{M}$ mit $\boldsymbol{N}$ und $\boldsymbol{M}^{\prime}$ mit $\boldsymbol{N}^{\prime}$ verbinden, ohne dazwischen durch den Anfangspunct zu gehen.

Ein ähnlicher Ausdruck, den man im Folgenden finden wird, nämlich

$$
u=\zeta \sqrt{1-\varkappa^{2} z^{2}}+z \sqrt{1-\gamma^{2} \zeta^{2}}=0
$$

stellt gleichfalls ein Lemniscatenstück dar. Behandelt man ihn wie den früheren, setzt also

$$
v=\zeta \sqrt{1-\varkappa^{2} \varepsilon^{2}} \quad z \sqrt{1-\gamma^{2} z^{2}}
$$

Journal f. d. M. Bd. LIII. Heft 3 . 
so findet man

$$
\frac{u v i}{2}=2 m n+h\left(m^{2}+n^{2}\right)^{2}=0
$$

oder die Polargleichung

$$
\rho=\sqrt{\frac{-\sin 2 \theta}{h}} .
$$

Lag $\varkappa$ im ersten Quadranten, so durchschneidet die Hauptaxe den zweiten und vierten Quadranten, sonst den ersten und dritten. Sie bildet mit einer Richtung der $\boldsymbol{X}$ Axe den Winkel $\frac{\pi}{4}$. Die $\approx$ desjenigen Theiles der Lemniscate, welcher $u=0$ entspricht, werden durch die Gleichung

$$
\boldsymbol{z}^{2}=\frac{1}{x^{2}-\mu^{2}}
$$

gegeben, wo $\mu$ irgend eine reelle Gröfse vorstellt, so dafs wieder zwei Lemniscaten-Stücke hierhergehören, welche in dem Punkte $\frac{1}{x}$ und $-\frac{1}{x}$ endigen, die aber in $\boldsymbol{A}$ beginnen. Es ist auch leicht zu sehen, welche von diesen Stücken zu nehmen sind. Es ist klar, dafs nicht für dasselbe z zugleich $\boldsymbol{u}$ und $r$ verschwinden, weil nicht ein reelles $m$ und $\mu$ existirt, für welche

$$
\frac{1+m^{2}}{1+x^{2} m^{2}}=\frac{1}{x^{2}-\mu^{2}}
$$

ist, weil sonst $\varkappa^{2}$ gleich der reellen Gröfse $1+\mu^{2}\left(1+m^{2}\right)$ wäre.

S. 14.

Wird an einer Stelle $p, q$ oder $r$ gleich 0 , so können sie dann ihr Zeichen ändern. Offenbar ändert $q$ sein Zeichen unter denselben Bedingungen

Fig. 2.

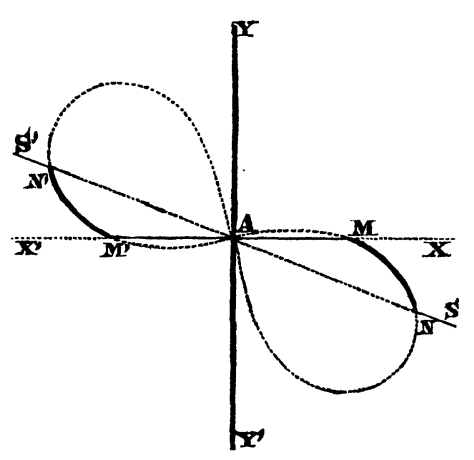
wie im $\$$. 8, nämlich wenn $z$ die Achse $\boldsymbol{Y}^{\prime} \boldsymbol{Y}$ schneidet, d. h. wenn $z$ rein imaginär wird, und der reelle Theil von $\approx$ sein Zeichen ändert; ferner auch wenn $\approx$ das Stück $\boldsymbol{M}^{\prime} \boldsymbol{M}$ durchschneidet, d. h. reell und $<1$ wird, und dabei sein imaginärer Theil das Zeichen wechselt. Es ändert $\boldsymbol{p}$ sein Zeichen, wenn $z$ die Linien $\mathbf{N} \mathbf{S}$ oder $\mathbf{N}^{\prime} \mathbf{S}^{\prime}$ durchschneidet, d. h. wenn der imaginäre Theil von $\varkappa z$ das Zeichen wechselt, und dabei $\varkappa z$ reell und $>1$ wird. Endlich wird $\boldsymbol{r}$ sein Zeichen ändern, wenn $\approx$ eines der Lernniscaten-Stücke $\boldsymbol{M N}$ oder $\boldsymbol{M}^{\prime} \boldsymbol{N}^{\prime}$ durchschneidet, was man vermittelst der Polargleichung des \$. 13 analytisch ausdrücken kann. 
Die letzlere Behauptung erweist man durch Betrachtung des Productes

$$
-\frac{r s i}{2}=2 m n(1-g)+h\left(\left(m^{2}+n^{2}\right)^{2}-\left(m^{2}-n^{2}\right)\right)
$$

das für $m$ und $n$ gleich $\infty$, also auch für jeden Punct aufserhalb der Lemniscate das Zeichen von $h$ hat; für jeden Punct der Linie selbst 0 ist; für den Punct im Innern $m=\frac{1}{2}, n=0$, also für alle innern Puncte das Zeichen von $-\boldsymbol{h}$ hat. Es ändert also $r$ s sein Zeichen, wenn $\approx$ die Stücke $M N$ und $\boldsymbol{M}^{\prime} \boldsymbol{N}^{\prime}$ durchschneidet und zwar dadurch, dafs $\boldsymbol{r}$ durch Null gebt, nicht dadurch dafs $s$ verschwindet. Es hat also $r$ das Zeichen geändert.

\$. 15.

Auf ganz ähnliche Art wie im $\$$. 8. untersuchen wir hier die Zeichen von

$$
\begin{aligned}
& \boldsymbol{A}=a \sqrt{1-a^{2}}\left(\sqrt{1-\varkappa^{2} a^{2}}+\sqrt{1-\gamma^{2} a^{2}}\right) \\
& \boldsymbol{B}=b \sqrt{1-b^{2}}\left(\sqrt{1-\chi_{1}^{2} b^{2}}+\sqrt{1-\gamma_{1}^{2} b^{2}}\right)
\end{aligned}
$$

und bilden deshalb Ausdrücke, die mit $\boldsymbol{A}$ und $\boldsymbol{B}$ gleiche Zeichen haben; die Gleichungen $(\delta$.$) und (\varepsilon$.$) des \$$. 10 werden hierzu jedoch nicht ausreichen, da in ihnen $\sqrt{1-\varkappa^{2} a^{2}}$ und $\sqrt{1-\varkappa_{1}^{2} b^{2}}$ nicht vorkommen. Man gehe deshalb auf (4.) zurück, dividire beide Seiten durch $z$, und nehme die reellen Theile. Benutzt man dann die Gleichung

$$
\sqrt{1-b^{2}}=\frac{p}{r} \sqrt{1-a^{2}}
$$

die aus $(\delta$.$) und (\varepsilon$.$) folgt, so wird$

$$
\boldsymbol{A z} \zeta=\frac{q i(z-\zeta)\left(1-a^{2}\right)+a^{2} r\left(\zeta \sqrt{1-x^{2} z^{2}}+z \sqrt{1-x^{2} \zeta^{2}}\right)}{p}
$$

erhalten. Eine zweite Formel, die wir hier anwenden, folgt aus (3.) und (4.) durch Elimination von $\sqrt{1-\varkappa^{2} z^{2}}$; daraus entsteht

$$
b \sqrt{1-\varkappa^{2}} a^{2}=\frac{i \sqrt{1-z^{2}}}{z}-\frac{i \sqrt{1-a^{2}} \sqrt{1-b^{2}}}{z} \text {. }
$$

Der reelle Theil der vorstehenden Gleichung giebt

$$
\begin{gathered}
\frac{b^{2} z \zeta\left(\sqrt{1-x^{2} a^{2}}+\sqrt{1-\gamma_{1}^{2} a^{2}}\right) a}{\sqrt{1-a^{2}}} \\
=-\frac{q i}{r}\left(\zeta \sqrt{1-z^{2}}-z \sqrt{1-\zeta^{2}}+\sqrt{1-a^{2}} \sqrt{1-b^{2}}(z-\zeta)\right) .
\end{gathered}
$$

Die obere Formel lehrt, dafs, da $a^{2}$ continuirlich ist, dasselbe für $\boldsymbol{A}$ gilt, vielleicht mit Ausnahme des Werthes $p=0$, für den (s. oben) $b$ nicht Null ist. Da für keinen Werth, der im Laufe von z vorkommt, $r$ zugleich Null wird, so sieht man aus der letzten Formel, daf's $\boldsymbol{A}$ auch dann noch con28 * 
tinuirlich bleibt; es bleibt sogar noch continuirlich für $p=r=0 ; d$. h. für $\varkappa z=1$. Es kann daher $A$ wiederum sein Zeichen nur bei $a^{2}=1$ oder 0 ändern. Da nun $a^{2}$ eine continuirliche Function von $z=m+n i$, oder von $m$ und $n$ ist, und 0 und 1 ihre kleinsten und gröfsten Werthe sind, so ist, auch ohne dafs die Rechnung ausgeführt wird, klar, dafs eine unendlich kleine Aenderung der ersten Ordnung von $z-$ sie sei $\varepsilon$ - an den betreffenden Stellen, eine Aenderung zweiter Ordnung, also von der Ordnung $\varepsilon^{2}$ für $a^{2}$ giebt. Es ist nun $a=0$ wenn $z$ rein imaginär $=n i$ wird; für diesen Fall hat man $\boldsymbol{A}=0$. Aendert sich $z$ dann $\mathrm{um} \varepsilon$, so ändert sich $q$ um ein Glied der Ordnung $\varepsilon ; z-\zeta$ und $1-a^{2}$ sind endliche Gröfsen, d. h. nicht $0 ; a^{2}$ ist von der Ordnung $\varepsilon^{2}$, so dafs $\frac{q i(z-\zeta)}{p}$ das Zeichen des neuen $\boldsymbol{A}$ bestimmt. Es ändert daher (\$. 14.) $\boldsymbol{A}$ sein Zeichen, wenn $\boldsymbol{z}$ die Achse des Imaginären durchschneidet. Wird $a^{2}=1$ also $r=0$, so ist $1-a^{2}=0$, aber weder $q$ noch $z-\zeta$ verschwindet. Da nun die Lemniscate $r=0$ die zweite

$$
\zeta \sqrt{1-\varkappa^{2} z^{2}}+z \sqrt{1-\gamma^{2} \zeta^{2}}=0
$$

(s. \$. 13.) nicht schneidet, der letzte Ausdruck daher für $r=0$ nicht verschwindet, so bestimmt das Zeichen von

$$
\frac{a^{2} r\left(\zeta \sqrt{1-x^{2} z^{2}}+z \sqrt{1-\gamma^{2} \zeta^{2}}\right)}{\prime \prime}
$$

das Zeichen von $\boldsymbol{A}$, so dafs $\boldsymbol{A}$ sein Zeichen wechselt, wenn $\boldsymbol{z}$ die LemniscatenStücke $M N$ und $M^{\prime} N^{\prime}$ durchschneidet.

Um das Zeichen von $B$ zu finden, dividire man (5.) durch $z$, und multiplicire mit $\frac{b}{\sqrt{1-b^{2}}}$; die Gleichsetzung der reellen Theile giebt dann

$$
z \zeta . b \cdot \frac{\sqrt{1-x_{1}^{2} b^{2}}+\sqrt{1-\gamma_{1}^{2} b^{2}}}{\sqrt{1-b^{2}}}=-\frac{(z+\zeta) q}{p}+\frac{i b^{2} r}{p}\left(\zeta \sqrt{1-\varkappa^{2} z^{2}}-z \sqrt{1-\gamma^{2} \zeta^{2}}\right),
$$

und nach Multiplication mit $1-b^{2}=\frac{a^{2} b^{2} p^{2}}{q^{2}}$ einen zweiten Ausdruck:

$$
\boldsymbol{z} \zeta \boldsymbol{B}=\frac{a^{2} b^{2} p}{q}\left(-q(z+\zeta)+i b^{2} r\left(\zeta \sqrt{1-\varkappa^{2} z^{2}}-z \sqrt{1-\gamma^{2} \zeta^{2}}\right)\right)
$$

Es bleibt daher auch $B$ continuirlich, ändert folglich sein Zeichen nur für $b^{2}=0$ und $b^{2}=1$, und zwar nur und immer, wenn $z$ die Stücke $M^{\prime} M$ $\operatorname{oder} N \mathbf{S}$ oder $\mathbf{N}^{\prime} \mathbf{S}^{\prime}$ durchschneidet.

\$. 16.

Wie im \$. 9. seien jetzt $\left[\boldsymbol{A}_{0}\right]$ und $\left[\boldsymbol{B}_{0}\right]$ die Anfangszeichen von $\boldsymbol{A}$ und $B$; wir folgen dem Laufe von $z$ und nennen den Zahlwerth des an- 
fänglichen $a$ wieder $\alpha_{0}$, des letzten $a$ gleichfalls $\mathfrak{A}$, die entsprechenden Stücke für $b, \beta_{0}$ und $\mathfrak{B}$. Die Zahlenwerthe der $a$ an den Stellen, an welchen die Curve $z$ die in der Figur stärker gezogenen Linien schneidet, heifsen der Reihe nach $\alpha_{1}, \alpha_{2}, \ldots \alpha_{m}$, so dafs beim Durchschnitt mit der Achse $\boldsymbol{Y}^{\prime} \boldsymbol{Y}$ immer $\alpha=0$, wenn $M N$ oder $M^{\prime} \boldsymbol{N}^{\prime}$ durchschnitten wird, $\alpha=1$ gesetzt wird. Ähnlich setzen wir $\beta=0$ beim Durchschnitt mit $\boldsymbol{M}^{\prime} \boldsymbol{M}, \beta=1$ beim Durchschnitt mit NS oder $\boldsymbol{N}^{\prime} \boldsymbol{S}^{\prime}$, und haben dann wieder für $\boldsymbol{J}$ die Formel (8.), in der $\sqrt{1-\alpha^{2}}, \sqrt{1-\beta^{2}}$ die positiven Fig. 2.

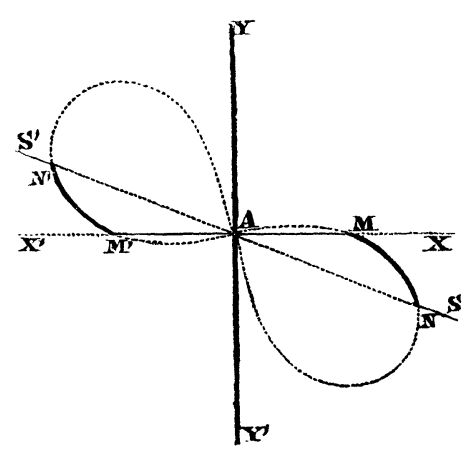
Wurzeln, $\sqrt{1-\varkappa^{2} \alpha^{2}}, \sqrt{1-\varkappa_{1}^{2} \beta^{2}}$ die mit positivem reellen Theile vorstellen.

Dem besondern, am Ende des $\$$. 9. angegebenen Falle entspricht das Resultat, welches bereits im Monatsbericht der Berliner Akademie 1855 p. 306 mitgetheilt wurde.

\section{\$. 17.}

Wird $\varkappa$ reell, so mufs (9.) und (10.) dieselben Werthe für $a$ und $b$ geben, welche man $\$$. 6 . findet. Da es einige Mühe zu machen scheint, dieses zu zeigen, so soll die Rechnung wenigstens für die eine Formel hier ausgeführt werden.

Es ist offenbar $2 p$ das Product der beiden Factoren

$$
\begin{aligned}
& f=\sqrt{1+x z} \sqrt{1+\gamma \zeta}+\sqrt{1-x z} \sqrt{1-\gamma \zeta} \\
& f_{1}=\sqrt{1-x z} \sqrt{1+\gamma \zeta}+\sqrt{1+x z} \sqrt{1-\gamma \zeta}
\end{aligned}
$$

und

wenn

$$
2\left(\sqrt{1-z^{2}}-\sqrt{1-\zeta^{2}}\right)=g g_{1}
$$

endlich

$$
\begin{aligned}
& g=\sqrt{1+z} \sqrt{1+\zeta}-\sqrt{1-z} \sqrt{1-\zeta} \\
& g_{1}=\sqrt{1-z} \sqrt{1+\zeta}-\sqrt{1+z} \sqrt{1 \zeta}
\end{aligned}
$$

so dafs (9.) in

$$
4\left(p^{2}-q^{2}-r^{2}\right)=f^{2} g_{1}^{2}+f_{1}^{2} g^{2}-4 z^{2} \zeta^{2}(\varkappa-\gamma)^{2},
$$

also für $x=\gamma$ in

$$
f^{2} f_{1}^{2} a^{4}-a^{2}\left(f g_{1}^{2}+f_{1}^{2} g^{2}-4 z^{2} \zeta^{2}(x-\gamma)^{2}\right)+g^{2} g_{1}^{2}=0
$$

$\left(a^{2} f^{2}-g^{2}\right)\left(a^{2} f_{1}^{2}-g_{1}^{2}\right)=0$
übergeht. Es ist daher $a^{2}=\frac{g^{2}}{f^{2}}$ oder $a^{2}=\frac{g_{1}^{2}}{f_{1}^{2}}$, von welchen Werthen aus naheliegenden Gründen jedoch nur der erste zu nehmen ist. 
§. 18.

Wir schliefsen diesen Theil mit einer Stelle aus einer Arbeit von Jacobi, Bd. 8, p. 416 und 417 dieses Journals, die wir der Vollständigkeit halber, mit ganz unwesentlichen Veränderungen hinzufügen.

Die elliptischen Integrale mit reellen Grenzen, welche kleiner als 1 sind, auf die wir im allgemeinen Falle $\boldsymbol{J}$ zurückgeführt haben, enthalten einen imaginären Modulus $\varkappa . J a c o b i$ hat die Substitution angegeben, durch welche ein solches Integral sich in die Form $\boldsymbol{M}+\boldsymbol{N i}$ zerlegen läfst, wenn $\boldsymbol{M}$ und $\boldsymbol{N}$ reelle $A b e l s c h e$ Integrale bezeichnen. Nach dem Obigen ist $J$ auf eine Summe oder Differenz von Integralen

$$
L=\int_{0}^{\eta} \frac{d x}{\sqrt{1-x^{2}} \sqrt{1-x^{2} x^{2}}}
$$

zurückgeführt, wo $\eta, \sqrt{1-x^{2}}$ und der reelle Theil von $\sqrt{1-\varkappa^{2} x^{2}}$ so wie von $\approx$ positiv sind. Setzt man

$$
\begin{aligned}
& \text { (14.) } \lambda=\left(\frac{x+\gamma}{x_{1}+\gamma_{1}}\right)^{2} \\
& \text { (15.) } \mu=-\left(\frac{x-\gamma}{x_{1}+\gamma_{1}}\right)^{2} \text {, }
\end{aligned}
$$

so dafs sowohl $\lambda$ als $\mu$ positiv sind, ferner $\varkappa=e+f i$, so ist $\mu<1$. Wendet man nämlich die Substitution des \$. 4. an, macht also

$$
\begin{aligned}
& 1=\rho \sin \theta \\
& f=\sqrt{\rho^{2}-e^{2}} \cos \theta,
\end{aligned}
$$

so wird

$$
\begin{aligned}
\varkappa & =e+i \cos \theta \sqrt{\varrho^{2}-e^{2}} \\
\varkappa_{1} & =\sqrt{\varrho^{2}-e^{2}}-i e \cos \theta,
\end{aligned}
$$

also

$$
\lambda=\frac{\rho^{2}}{\rho^{2}-e^{2}} \quad \mu=\cos ^{2} \theta
$$

d. h. $\mu<1$. Führt man durch die Substition

$$
x=\sqrt{\frac{(1+\lambda)(1-\mu) z}{(1+\lambda z)(1-\mu z)}}
$$

statt $x$ die Gröfse $z$ ein, indem man die Wurzel positiv nimmt, so wird $z$ reell und $<1$ sein. Man findet nämlich für $z$ die Gleichung

$$
\lambda \mu z^{2}-z\left(\lambda-\mu-\frac{(1+\lambda)(1-\mu)}{x^{2}}\right)-1=0 ;
$$


für $z=0$ und $z=1$ ist aber die linke Seite resp. -1 und positiv, weil sie selbst im schlimmsten Falle, d. h. für $x=1$ nicht negativ wird. Ein Werth von $z$ ist also positiv und kleiner als 1 ; es mag für $x=\eta$ der Werth von $z$ gleich $\zeta$ sein.

Es wird nun

$$
\begin{aligned}
\sqrt{1-x^{2}} & =\sqrt{\frac{(1-z)(1+\lambda \mu z)}{(1+\lambda z)(1-\mu z)}} \\
\sqrt{1-\varkappa^{2} x^{2}} & =\frac{1 \mp i z \sqrt{\lambda \mu}}{\sqrt{(1+\lambda z)(1-\mu z)}}
\end{aligned}
$$

wo die oberen oder die unteren Zeichen zu nehmen sind, je nachdem $f$; der imaginäre Theil von $\varkappa$, positiv oder negativ ist. Ferner ist

also

$$
d x=\frac{1}{2} \sqrt{1+\lambda} \sqrt{1-\mu} \frac{(1-\lambda \mu z) d z}{\sqrt{z}(1+\lambda z)^{\frac{3}{2}}(1-\mu z)^{\frac{3}{2}}}
$$

wenn

$$
\boldsymbol{L}=\boldsymbol{M} \pm \boldsymbol{N} i
$$

$$
\begin{gathered}
M=\frac{1}{2} \sqrt{1+\lambda} \sqrt{1-\mu} \int_{0}^{\zeta} \frac{d z}{\sqrt{z \sqrt{1-z}} \sqrt{1+\lambda z} \sqrt{1-\mu z} \sqrt{1+\lambda \mu z}} \\
N=\frac{1}{2} \sqrt{1+\lambda} \sqrt{1-\mu} \sqrt{\lambda \mu} \int_{0}^{\zeta} \frac{\sqrt{z} \cdot d z}{\sqrt{1-z} \sqrt{1+\lambda z} \sqrt{1-\mu z} \sqrt{1+\lambda \mu z}}
\end{gathered}
$$

gesetzt wird.

\section{Abtheilung.}

S. 19.

Nach den Untersuchungen im ersten Theile ist es leicht, das Integral

$$
\boldsymbol{H}=\int_{g}^{h} \frac{d x}{\sqrt{(x-a)^{2}+\bar{b}^{2}} \sqrt{(x-\alpha)^{2}+\beta^{2}}}
$$

in die Summe eines reellen und imaginären Theiles zu zerlegen. Es bezeichnen hier $a, b, \alpha, \beta$ reelle oder imaginäre Constante; unter $g$ und $h$ sollen reelle Grenzen verstanden werden, zwischen denen $x$ sich reell bewegt. Würde $x$ auf gegebne Art zwischen irgend welchen Grenzen sich bewegen, ohne dals es fortwährend reell zu sein brauchte, so würde unsere Methode sich unmittelbar auf diesen Fall übertragen lassen. Wir denken uns, dafs die Quadratwurzeln sich continuirlich ändern, und dafs die Zeichen derselben für eine Anzahl von Werthen von $x$ gegeben sind, die hinreichend ist, um sie für 
jedes $x$ zu bestimmen. Verschwindet z. B. keine Wurzel, so genŭgt die Angabe der Zeichen für ein $x$. (M. vergl. \$. 3.)

Man setze nun

$$
\varkappa=\sqrt{\frac{(a-\alpha)^{2}+(b-\beta)^{2}}{(a-\alpha)^{2}+(b+\beta)^{2}}}
$$

indem man unter $b$ und $\beta$ solche Wurzeln aus $b^{2}$ und $\beta^{2}$ versteht, welche den Modulus von $\varkappa$ kleiner als 1 machen, und die Wurzel so nimmt, dafs ihr reeller Theil positiv ist. Ferner setze man

$$
\boldsymbol{M}=\sqrt{(\boldsymbol{a}-\boldsymbol{\alpha})^{2}+(\boldsymbol{b}+\boldsymbol{\beta})^{2}},
$$

das Zeichen der Wurzel beliebig genommen, und führe durch die Gleichung

$$
\frac{1-x z}{1+x z}=\frac{b}{\beta} \frac{(x-\alpha)^{2}+\beta^{2}}{(x-a)^{2}+b^{2}}
$$

oder, was dasselbe ist, durch

$$
x z=\frac{\beta\left((x-a)^{2}+b^{2}\right)-b\left((x-\alpha)^{2}+\beta^{2}\right)}{\beta\left((x-a)^{2}+b^{2}\right)+b\left((x-\alpha)^{2}+\beta^{2}\right)}
$$

eine neue Veränderliche $z$ ein. Hierdurch ist $z$, ebenso $1-z^{2}$ und $1-x^{2} z^{2}$ bestimmt; unter den Wurzeln aus den letzten Gröfsen sollen folgende Ausdrücke mit bekanntem Zeichen verstanden werden:

$$
\begin{aligned}
\sqrt{1-z^{2}} & =\frac{2 \sqrt{b \beta}}{x M} \frac{\left((x-\alpha)^{2}+\beta^{2}\right)(x-a)-\left((x-a)^{2}+b^{2}\right)(x-\alpha)}{\beta\left((x-a)^{2}+b^{2}\right)+b\left((x-\alpha)^{2}+\beta^{2}\right)} \\
\sqrt{1-x^{2} z^{2}} & =\frac{2 \sqrt{b \beta} \sqrt{(x-a)^{2}+b^{2}} \sqrt{(x-\alpha)^{2}+\beta^{2}}}{\beta\left((x-a)^{2}+b^{2}\right)+b\left((x-\alpha)^{2}+\beta^{2}\right)},
\end{aligned}
$$

wenn $\sqrt{b \beta}$ eine willkürliche, aber feste Quadratwurzel aus $b \beta$ ist, und die übrigen Quadratwurzeln die früher beslimmten Zeichen haben. Dann wird

$$
\boldsymbol{H}=\frac{1}{M} \int_{g}^{h /} \frac{z^{\prime} d x}{\sqrt{1-z^{2}} \sqrt{1-x^{2} z^{2}}}=\frac{1}{M} \cdot J
$$

Sollte $\varkappa$ eine rein imaginäre Gröfse geworden sein, so kann man durch die Substitution

$$
y=\sqrt{1-z^{2}}
$$

$\boldsymbol{J}$ auf ein ähnliches Integral mit einem neuen Modulus $\varkappa$ zurückführen, der reell und $<1$ ist; natürlich ist es nicht nöthig, zweimal zu substituiren; man kann gleich für $x$ die Gröfse $y$ einführen. $J$ zerlegt man nun nach den Regeln des ersten Abschnittes. 
§. 20.

So lange $a, b, \alpha, \beta$, allgemein bleiben, gelang es uns nicht, in den Endformeln wesentliche Vereinfachungen vorzunehmen; die Berechnung aller hierher gehörigen Werthe bietet offenbar keine anderen Schwierigkeiten dar, als die Länge der Rechnung. Ein besonderer Fall, auf den ich bei der Lösung einer Aufgabe aus der Theorie der Anziehung geführt wurde, lälst eine wesentliche Vereinfachung zu, und soll hier als Beispiel behandelt werden; es ist dies der Fall

$$
\text { (16.) } \quad \boldsymbol{H}=\int_{0}^{1} \frac{d x}{\sqrt{(x-m i)^{2}-n^{2}} \sqrt{(x-\mu i)^{2}-v^{2}}},
$$

wenn $m, n, \mu, \nu$ reelle, $n$ und $\nu$ positive Gröfsen bezeichnen. Die Wurzeln sollen sich continuirlich ändern, und so beschaffen sein, dafs ihr reeller Theil, wo ein solcher zuerst auftritt, positiv ist, woraus zugleich folgt, dafs der reelle Theil für jedes $x$ aufser 0 positiv bleibt.

In Folge des $\$$. 19. setzen wir

$$
\mathfrak{M}=\sqrt{(\boldsymbol{n}-\boldsymbol{\mu})^{2}+(\boldsymbol{n}+\boldsymbol{v})^{2}},
$$

die Wurzel positiv genommen, und $M=i \mathfrak{M}$, ferner die positive, reelle Gröfse $\varkappa$

dann

$$
\text { (17.) } \quad x=\sqrt{\frac{(m-\mu)^{2}+(n-\nu)^{2}}{(m-\mu)^{2}+(n+\nu)^{2}}} \text {, }
$$

$$
\begin{aligned}
& \text { (18.) } \quad \varkappa=\frac{\nu\left((x-m i)^{2}-n^{2}\right)-n\left((x-\mu i)^{2}-\nu^{2}\right)}{\nu\left((x-m i)^{2}-n^{2}\right)+n\left((x-\mu i)^{2}-\nu^{2}\right)} \\
& \text { (19.) } \sqrt{1-z^{2}}=\frac{2 i \sqrt{n \nu}}{x \mathfrak{M}} \frac{\left((x-m i)^{2}-n^{2}\right)(x-\mu i)-\left((x-\mu i)^{2}-v^{2}\right)(x-m i)}{\nu\left((x-m i)^{2}-n^{2}\right)+n\left((x-\mu i)^{2}-\nu^{2}\right)} \\
& \text { (20.) } \sqrt{1-\varkappa^{2} s^{2}}=2 \sqrt{n \nu} \frac{\sqrt{(x-m i)^{2}-n^{2}} \sqrt{(x-\mu i)^{2}-\nu^{2}}}{\nu\left((x-m i)^{2}-n^{2}\right)+n\left((x-\mu i)^{2}-\nu^{2}\right)}
\end{aligned}
$$

wo die $\sqrt{\boldsymbol{n} \nu}$ positiv genommen wird. Wir fügen noch die zwei Formeln

$$
\text { (21.) } \frac{1-x z}{1+x z}=\frac{n}{v} \frac{(x-\mu i)^{2}-v^{2}}{(x-m i)^{2}-n^{2}}
$$

und

(22.) $x z^{\prime}=-4 n \nu \frac{\left((x-m i)^{2}-n^{2}\right)(x-\mu i)-\left((x-\mu i)^{2}-\nu^{2}\right)(x-m i)}{\nu\left((x-m i)^{2}-n^{2}\right)+n\left((x-\mu i)^{2}-\nu^{2}\right)}$

hinzu. Es wird dann endlich

$$
\text { (23.) } \quad \boldsymbol{H}=-\frac{i}{\mathfrak{M}} \cdot \int_{0}^{1} \frac{z^{\prime} d z}{\sqrt{1-z^{2}} \sqrt{1-x^{2} z^{2}}}=-\frac{i}{\mathfrak{M}} J
$$

Journal f. d. M. Bd. LIII. Heft 3. 
§. 21.

Um $\boldsymbol{J}$ zu zerlegen, führe man nach $\mathfrak{\$}$.4. Polarcoordinaten $\varrho, \sigma, \eta, \theta$ durch die Gleichungen

$$
\begin{array}{ll}
m=\sqrt{\varrho^{2}-1} \cos \eta & \mu=\sqrt{\sigma^{2}-1} \cos \theta \\
n=\varrho \sin \eta & \nu=\sigma \sin \theta
\end{array}
$$

ein; der Kürze halber sollen jedoch neben $\rho, \sigma$, etc. auch $m$, $n$, etc. in den Formeln gebraucht werden. Ferner sollen $\varepsilon$ und $\zeta$ gleich +1 oder -1 sein, je nachdem resp. $m$ oder $\mu$ positiv oder negativ sind. Dann wird

$$
\begin{aligned}
& \sqrt{(1-i m)^{2}-n^{2}}=\varepsilon\left(\cos \eta-i \sqrt{\varrho^{2}-1}\right) \\
& \sqrt{(1-i \mu)^{2}-v^{2}}=\zeta\left(\cos \theta-i \sqrt{\sigma^{2}-1}\right) .
\end{aligned}
$$

Die ziemlich mühsame Berechnung von $\mathfrak{A}$ und $\mathfrak{B}$ soll hier nicht ausgeführt werden ( $(9.9$.$) ; wir drückten zunächst \frac{\mathfrak{B}^{2}}{1-\mathfrak{B}^{2}}$ durch die bekannten Gröfsen nach \$. 6. aus, und führten in dem Zähler erst an einer gewissen Stelle, nachdem ein Factor desselben sich gehoben hatte, die Polarcoordinaten ein. Ist $\mathfrak{B}$ gefunden, so ergiebt sich $\mathfrak{A}$ ohne Wiederholung der Rechnung indem $\frac{\mathfrak{A}}{\sqrt{1-x_{1}^{2}} \overline{\mathcal{B}^{2}}}$ gleich dem Werthe von

$$
\frac{1}{x} \frac{\sqrt{1+x z} \sqrt{1+x \zeta}-\sqrt{1-x z} \sqrt{1-x \zeta}}{\sqrt{1+x z} \sqrt{1+x \zeta}+\sqrt{1-x z} \sqrt{1-x \zeta}}
$$

für $x=1$ ist. So findet man

$$
\begin{aligned}
& \text { (24.) } \quad x \mathfrak{A}=v \cdot n \cdot\left(\frac{\varrho \sin \theta-\sigma \sin \eta}{\varrho \sin \theta+\sigma \sin \eta}\right) \\
& \text { (25.) } \quad \mathfrak{B}=\frac{\sqrt{(m-\mu)^{2}+(n+\nu)^{2}}}{\varrho \sigma+\sin \theta \sin \eta}
\end{aligned}
$$

oder

$$
\frac{\mathfrak{B}}{\sqrt{1-\mathfrak{B}^{2}}}=\frac{\sqrt{(m-\mu)^{2}+(n+\nu)^{2}}}{\sqrt{\varrho^{2}-1} \sqrt{\sigma^{2}-1}+\cos \theta \cos \eta}
$$

Endlich ist

$$
\text { (26.) } \quad x \alpha_{0}=\text { v.n. }\left(\frac{\nu\left(m^{2}+n^{2}\right)-n\left(\mu^{2}+\nu^{2}\right)}{\nu\left(m^{2}+n^{2}\right)+n\left(\mu^{2}+\nu^{2}\right)}\right) ; \quad \beta_{0}=0 \text {. }
$$

Die Buchstaben $v . n$. bedeuten hier, dafs der Zahlwerth des betreffenden Ausdrucks genommen werden soll. 
\$. 22.

Um die Anfangszeichen $\left[\boldsymbol{A}_{0}\right]$ und $\left[\boldsymbol{B}_{0}\right]$ zu finden, setzen wir in die betreffenden Formeln des $\$$. 8. den Werth $z=0$. Für $\left[\boldsymbol{A}_{1}\right]$ hat diese Rechnung keine Schwierigkeit, während die Untersuchung für $\left[\boldsymbol{B}_{0}\right]$ die Kenntnifs des Anfangszeichens von $\frac{z-\zeta}{i}$ erfordert, das man jedoch leicht mit Benutzung der Formel (22.) für $z^{\prime}$ erkennt. Setzt man zur Abkürzung

so wird

$$
\begin{aligned}
\boldsymbol{R} & =\boldsymbol{\mu}\left(\boldsymbol{m}^{2}+\boldsymbol{n}^{2}\right)-\boldsymbol{m}\left(\boldsymbol{\mu}^{2}+\boldsymbol{\nu}^{2}\right) \\
\boldsymbol{S} & =\boldsymbol{\nu}\left(\boldsymbol{m}^{2}+\boldsymbol{n}^{2}\right)-\boldsymbol{n}\left(\boldsymbol{\mu}^{2}+\boldsymbol{\nu}^{2}\right),
\end{aligned}
$$

$$
\begin{aligned}
& {\left[\boldsymbol{A}_{0}\right]=\varepsilon \zeta[\boldsymbol{R S}]} \\
& {\left[\boldsymbol{B}_{0}\right]=-\varepsilon \zeta .}
\end{aligned}
$$

\$. 23.

Es sind endlich die Stellen aufzusuchen, an denen z reell oder rein imaginär wird, um zu erfabren, wo $\boldsymbol{A}$ oder $\boldsymbol{B}$ sein Zeichen ändert.

Es ist $z$ mit $\frac{1-x z}{1+x z}$ also nach (21.) mit

$$
\frac{(x-\mu i)^{2}-\nu^{2}}{(x-m i)^{2}-n^{2}}
$$

zugleich reell. Setzt man den letzten Ausdruck $=v$, so findet man zwei Gleichungen, die stattfinden müssen, wenn $v$ reell sein soll, von denen die eine den Werth von $v$, die andere den entsprechenden von $x$ liefert, nämlich

$$
x^{2}=\frac{\boldsymbol{R}}{\mu-m} .
$$

Ist also $\frac{R}{\mu-m}$ positiv und $<1$, so wird, während $x$ von 0 bis 1 wächst, $z$ für einen und nur einen Werth von $x$ reell sein. Man weifs aus $\$$. 8 , dafs es nothwendig ist $\mathrm{zu}$ untersuchen, ob dieses reelle $z$ zwischen 0 und 1 , zwischen 1 und $\frac{1}{x}$ oder über $\frac{1}{x}$ hinaus liegt. Man findet aber für dasselbe die Gleichung

so dafs

$$
x z=\frac{m \nu-\mu n}{m \nu+\mu n}
$$

$$
1-x^{2} z^{2}=\frac{4 m n \mu \nu}{(m \nu+\mu n)^{2}}
$$


also positiv wird, wenn $\varepsilon=\zeta$, sonst negativ; d. h. es ist $z>\frac{1}{x}$ wenn $\varepsilon=-\zeta$. Man wird unten sehen, dafs für $\varepsilon=-\zeta$ immer $\frac{\boldsymbol{R}}{\mu-m}$ gröfser als 1 ist, so dafs niemals $z>\frac{1}{x}$ werden kann. Ist $\varepsilon=\zeta$, so wird jedenfalls $z>1$, weil nach (19.)

also imaginär ist.

$$
\sqrt{1-z^{2}}=\frac{2 i \sqrt{n \nu} x}{x \mathfrak{M}(m \nu+\mu)}
$$

Soll zweitens z rein imaginär sein, so mufs die rechte Seite von (21.) durch Vertauschung von $i$ mit $-i$ in $\frac{1+x z}{1-x z}$ übergehen, woraus sich der betreffende Werth von $x$, nämlich

$$
x^{2}=\frac{s}{n-v}
$$

berechnen läfst. Denkt man sich $n>\nu$, so wird also $x$ reell, wenn $\boldsymbol{S}$ positiv ist, und $z$ wird innerhalb der Integrationsgrenzen rein imaginär, wenn aufserdem $\frac{S}{n-v}<1$.

\section{\$. 24.}

Über die Zeichen und Werthe der hier vorkommenden Gröfsen kann man noch Folgendes bemerken:

1. Da $n>v$ sein sollte, so ist $\left[\frac{S}{n-\nu}\right]=[S]$.

2. Ist $\varepsilon=-\zeta$, so ist $\left[\frac{R}{\mu-m}\right]=+1 ;[R]=\zeta$.

3. Führt man die Polarcoordinaten ein, so ist

$$
\frac{\boldsymbol{R}}{\mu-m}=1+\frac{\sqrt{\sigma^{2}-1} \cos \eta-\sqrt{\rho^{2}-1} \cos \theta}{\sqrt{\rho^{2}-1} \cos \eta-\sqrt{\sigma^{2}-1} \cos \theta}\left(\sqrt{\rho^{2}-1} \sqrt{\sigma^{2}-1}+\cos \eta \cos \theta\right)
$$

also sicher gröfser als 1 , wenn $\varepsilon=-\zeta$ ist.

4. Ebenso findet man

$$
\frac{s}{n-\nu}=1+\frac{\rho \sin \theta-\sigma \sin \eta}{\rho \sin \eta-\sigma \sin \theta}(\rho \sigma-\sin \theta \sin \eta)
$$

wird der Ausdruck, dessen Zahlwerth $\mathfrak{A}$ ist, positiv, so ist also sicher zugleich $\frac{S}{n-\nu}>1$ und positiv; soll $\frac{s}{n-\nu}<1$ sein, so ist jene Gröfse negativ.

5. Jene Gröfse, deren Zahlwerth $\alpha_{10}$ ist, hat das Zeichen von $\mathbf{S}$. 


\section{S. 25.}

Man kann nun $J=u+i v$ setzen, und findet nach (8.) folgende Ausdrücke für $u$ und $v$, die sich übrigens durch die Bemerkungen des vorigen Paragraphen noch etwas vereinfachen lassen:

$$
v=-\varepsilon \zeta \int_{0}^{p} \frac{d \beta}{\sqrt{1-\beta^{2}} \sqrt{1-x_{1}^{2} \beta^{2}}} .
$$

Zweitens geht $u$, je nach den verschiedenen unten aufgezeichneten Fällen, in nachstehende Ausdrücke über, in denen die Function unter dem Integralzeichen immer

ist.

$$
\frac{d \alpha}{\sqrt{1-\alpha^{2}} \sqrt{1-x^{2} \alpha^{2}}}
$$

1. Es sei $\frac{S}{n-\nu}$ nicht zugleich positiv und $<1 ; \frac{R}{\mu-m}$ ebenso, so wird

$$
\boldsymbol{u}=\varepsilon \boldsymbol{\zeta}[\boldsymbol{R} \boldsymbol{S}] \int_{\boldsymbol{\alpha}_{n}}^{\mathfrak{U}}
$$

2. $\frac{S}{n-\nu}$ positiv und $<1 ; \frac{R}{\mu-m}$ wie ad 1 .

$$
\boldsymbol{u}=\varepsilon \zeta[\boldsymbol{R S}]\left(\int_{\boldsymbol{\alpha}_{0}}^{0}-\int_{0}^{\mathfrak{x}}\right)
$$

3. $\frac{S}{n-\nu}$ wie ad $1 ; \frac{R}{\mu-m}$ positiv und $<1$, (also auch $\varepsilon=\zeta$ )

$$
\boldsymbol{u}=\varepsilon ?[\boldsymbol{R S}]\left(\int_{\alpha_{0}}^{1}-\int_{1}^{\mathfrak{x}}\right)
$$

4. $\frac{S}{n-\nu}$ sowohl wie $\frac{R}{\mu-m}$ positiv und $<1$, (also auch $\varepsilon=\zeta$ )

a. $\frac{S}{n-\nu}$ ist kleiner als $\frac{R}{\mu-m}$

$$
u=\varepsilon \zeta[\boldsymbol{R S}]\left(\int_{\alpha_{0}}^{0}-\int_{0}^{1}+\int_{1}^{2 l}\right)
$$

ß. $\frac{S}{n-\nu}$ ist gröfser als $\frac{R}{\mu-m}$

$$
u=\varepsilon \zeta[R S]\left(\int_{\alpha_{0}}^{1}-\int_{1}^{0}+\int_{0}^{m}\right) .
$$

Es ist endlich

$$
\boldsymbol{H}=\frac{v}{\mathfrak{M}}-\frac{i \boldsymbol{u}}{\mathfrak{M}}
$$


S. 26.

Im Monatsberichte der Berliner Akademie 1854 S. 570 war die Aufgabe zu lösen,

$$
H_{1}=\int_{-1}^{1} \frac{d x}{N P \sqrt{1-\frac{n^{2}}{N^{2}}} \sqrt{1-\frac{\nu^{2}}{P^{2}}}}
$$

in die kanonische Form der elliptischen Integrale zu bringen, wenn

$$
\begin{aligned}
& \boldsymbol{N}=(\boldsymbol{x}-\boldsymbol{i m}) \\
& \boldsymbol{P}=(\boldsymbol{x}-\boldsymbol{i} \boldsymbol{\mu})
\end{aligned}
$$

ist, und die Quadratwurzeln so genommen werden, dafs ihr reeller Theil positiv bleibt. Zunächst ist klar, dafs dies Integral gleich dem doppelten reellen Theile des Integrales ist, welches sich von $\boldsymbol{H}_{1}$ nur dadurch unterscheidet, dafs seine Grenzen 0 und 1 sind. Um dieses auf die Form von $\boldsymbol{H}$ in $\mathfrak{\$} .20$. zu bringen, hat man zu untersuchen, ob

$$
N \sqrt{1-\frac{n^{2}}{N^{2}}}= \pm \sqrt{\mathbf{N}^{2}-n^{2}}
$$

wenn hier wie in dem ganzen Paragraphen die Wurzelzeichen so zu verstehen sind, dafs der reelle Theil positiv ist. Macht man für diese Untersuchung

$$
\begin{aligned}
\boldsymbol{m} & =\sqrt{\varrho^{2}-x^{2}} \cos \eta \\
\boldsymbol{n} & =\rho \sin \eta
\end{aligned}
$$

(wo also $\varrho$ und $\eta$ für jedes $x$ andere werden), und setzt $\varepsilon= \pm 1$, je nachdem $m$ positiv oder negativ ist, so wird

$$
\sqrt{\mathbf{N}^{2}-n^{2}}=\varepsilon\left(x \cos \eta-i \sqrt{\varrho^{2}-x^{2}}\right) .
$$

Ferner ist

$$
\frac{1}{x-m i}=\frac{x+i \sqrt{\varrho^{2}-x^{2}} \cos \eta}{x^{2} \sin ^{2} \eta+\rho^{2}}
$$

also der reelle Theil von $\frac{\sqrt{\bar{N}^{2}-n^{2}}}{x-m i}$ gleich

$$
\frac{\varepsilon \rho^{2} \cos \eta}{x^{2} \sin ^{2} \eta+\rho^{2}}
$$

also positiv, folglich $\frac{\sqrt{N^{2}-n^{2}}}{x-m i}=\sqrt{1-\frac{n^{2}}{N^{2}}}$. Es wird daher $H_{1}$ gleich dem doppelten reellen Theile von $H$, also

$$
H_{1}=-\frac{2 \varepsilon \zeta}{\mathfrak{R}} \int_{0}^{\mathfrak{B}} \frac{d \beta}{\sqrt{1-\beta^{2}} \sqrt{1-x^{2} \beta^{2}}}
$$


12. Heine, Reduktion elliptischer Integrale.

wenn $\varepsilon, \zeta, \mathfrak{M}, x$ dieselben Gröfsen wie im $\$ .21$ sind; d. h. es ist

$$
\begin{array}{rlrl}
m & =\sqrt{\varrho^{2}-1} \cos \eta & & \mu=\sqrt{\sigma^{2}-1} \cos \theta \\
n & =\varrho \sin \eta & \nu & =\sigma \sin \theta \\
\varepsilon . m & =v \cdot n \cdot(m) & \zeta \cdot \mu=v \cdot n \cdot(\mu) \\
\mathfrak{M} & =\sqrt{(m-\mu)^{2}+(n+\nu)^{2}} \\
\varkappa & =\frac{\sqrt{(m-\mu)^{2}+(n-\nu)^{2}}}{\mathfrak{M}} \\
\mathfrak{B} & =\frac{\mathfrak{M}}{\rho \sigma+\sin \theta \sin \eta} .
\end{array}
$$

\section{S. 27.}

In den allgemeinen Ausdrücken des $\$$. 19. kommen Quadratwurzeln aus Gröfsen wie $(x-a)^{2}+b^{2}$ vor, wo $a$ und $b$ selbst imaginär sind, während $x$ eine reelle Gröfse bezeichnet. Durch eine Transformationsformel, welche den im Vorhergehenden angewandten ähnlich ist, kann man auch solche Quadratwurzeln darstellen. Denkt man sich nämlich $a$ und $b$ in die Form

$$
\begin{aligned}
& a=e+f i \\
& b=g+h i
\end{aligned}
$$

zerlegt, so setze man

$$
\begin{aligned}
& e=\rho \sigma \cos \varphi \cos \psi+\sqrt{\varrho^{2}-x^{2}} \sqrt{\sigma^{2}-1} \sin \varphi \sin \psi \\
& f=\sigma \sqrt{\varrho^{2}-x^{2}} \sin \psi \cos \varphi-\varrho \sqrt{\sigma^{2}-1} \sin \varphi \cos \psi \\
& g=\sigma \sqrt{\varrho^{2}-x^{2}} \sin \varphi \cos \psi-\varrho \sqrt{\sigma^{2}-1} \sin \psi \cos \varphi \\
& h=\rho \sigma \sin \varphi \sin \psi+\sqrt{\varrho^{2}-x^{2}} \sqrt{\sigma^{2}-1} \cos \varphi \cos \psi
\end{aligned}
$$

und findet dann

$$
\sqrt{(x-a)^{2}+b^{2}}= \pm\left\{\rho \cos \psi-\sigma x \cos \varphi+i\left(\sqrt{\varrho^{2}-x^{2}} \sin \psi+x \sqrt{\sigma^{2}-1} \sin \varphi\right)\right\} \text {. }
$$

Nimmt man $\varrho, \sigma, \sqrt{\varrho^{2}-x^{2}}, \sqrt{\sigma^{2}-1}$ positiv, $\varrho>x, \sigma>1$, so sind alle Gröfsen bestimmt. Man hat nämlich :

$$
\begin{aligned}
e+h & =\left(\rho \sigma+\sqrt{\rho^{2}-x^{2}} \sqrt{\sigma^{2}-1}\right) \cos (\psi-\varphi) \\
e-h & =\left(\rho \sigma-\sqrt{\rho^{2}-x^{2}} \sqrt{\sigma^{2}-1}\right) \cos (\psi+\varphi) \\
f+g & =\left(\sigma \sqrt{\rho^{2}-x^{2}}-\rho \sqrt{\sigma^{2}-1}\right) \sin (\psi+\varphi) \\
f-g & =\left(\sigma \sqrt{\rho^{2}-x^{2}}+\rho \sqrt{\sigma^{2}-1}\right) \sin (\psi-\varphi),
\end{aligned}
$$


woraus z. B. für $\cos (\psi-\varphi)$ und $\cos (\psi+\varphi)$ die Bestimmung

$$
\begin{aligned}
& \frac{(e+h)^{2}}{\cos ^{2}(\psi-\varphi)}-\frac{(f-g)^{2}}{\sin ^{2}(\psi-\varphi)}=x^{2} \\
& \frac{(e-h)^{2}}{\cos ^{2}(\psi+\varphi)}-\frac{(f+g)^{2}}{\sin ^{2}(\psi+\varphi)}=x^{2}
\end{aligned}
$$

folgt, so dafs sich $x \cos (\psi-\varphi)$ und $x \cos (\psi+\varphi)$ geometrisch als Achsen von Hyperbeln ergeben. Ihre Zeichen findet man aus den vorstehenden Beziehungen zu $e \pm h$ etc., indem $\rho \sigma+\sqrt{\varrho^{2}-x^{2}} \sqrt{\sigma^{2}-1}, \sigma \sqrt{\rho^{2}-x^{2}}+\rho \sqrt{\sigma^{2}-1}$ positiv zu nehmen sind. Auf ähnliche Weise sind $\varrho$ und $\sigma$ Achsen von Ellipsen.

Bonn, im December 1855. 